\title{
金属氧胺化合物的结构、合成及电化学储能应用
}

\author{
赵 伟 ${ }^{1,2}$, 徐 阳 ${ }^{1,2}$, 万颖杰 ${ }^{1,2}$, 蔡天逊 ${ }^{1,2}$, 穆金潇 ${ }^{1,2}$, 黄富强 ${ }^{1,2}$
}

(1. 中国科学院 上海硅酸盐研究所, 高性能陶瓷和超微结构国家重点实验室, 上海 200050; 2 . 中国科学院大学 材料科学与光电技术学院, 北京 100049)

摘 要: 金属氧胺化合物 $\mathrm{M}_{x}(\mathrm{NCN})_{y}$ 作为类氧硫族化合物，是一类新兴的无机功能材料。准线性 $[\mathrm{NCN}]^{2-}$ 阴离子赋予 其空旷和具有孔道的晶体结构、独特的电子结构和新奇的物化性质，金属氧胺化合物在固态发光、光/电催化及电 化学储能等诸多领域展现出应用前景, 近年来逐渐成为研究热点。本文简要回顾了金属氧胺化合物的研究历史, 概 述了金属氰胺化合物的晶体结构及物化性质，总结了常见合成方法及策略，探讨了金属氧胺化合物在电化学储能 领域的应用，重点论述了其作为锂钠离子电池新型负极材料的电化学性能及存储机制。

关＼cjkstart键＼cjkstart词：金属氧胺化合物；晶体结构；合成方法；电化学储能；锂钠离子电池；综述

中图分类号: TQ174 文献标志码: A

\section{Metal Cyanamides/Carbodiimides: Structure, Synthesis and Electrochemical Energy Storage Performance}

\author{
ZHAO Wei $^{1,2}$, XU Yang ${ }^{1,2}$, WAN Yingjie ${ }^{1,2}$, CAI Tianxun ${ }^{1,2}$, MU Jinxiao ${ }^{1,2}$, HUANG Fuqiang ${ }^{1,2}$ \\ (1. State Key Laboratory of High Performance Ceramics and Superfine Microstructure, Shanghai Institute of Ceramics, Chinese \\ Academy of Sciences, Shanghai 200050, China; 2. Center of Materials Science and Optoelectronics Engineering, University of \\ Chinese Academy of Sciences, Beijing 100049, China)
}

\begin{abstract}
Metal cyanamides/carbodiimides $\left[\mathrm{M}_{x}(\mathrm{NCN})_{y}\right]$, as oxygen/chalcogenide-like compounds, are a new class of inorganic functional materials. The quasi-linear $[\mathrm{NCN}]^{2-}$ anion endows their open and porous crystal structure, unique electronic structure and unique physicochemical properties. They have shown potential applications in many fields including solid-state luminescence, photo/electrocatalysis, and electrochemical energy storage, becoming a research hotspot in recent years. This review outlines the research history of metal cyanamides, introduces the crystal structures and physicochemical properties, summarizes their synthetic methods and strategies, and discusses the applications for electrochemical energy storage, focusing on the electrochemical performance and charge storage mechanism as new-type negative electrode materials for lithium/sodium ion batteries.
\end{abstract}

Key words: metal cyanamides/carbodiimides; crystal structure; synthetic method; electrochemical energy storage; lithium/sodium ion battery; review

收稿日期：2021-09-24; 收到修改稿日期：2021-10-12; 网络出版日期：2021-10-21

基金项目：上海市自然科学基金(21ZR1473300)

Science and Technology Commission of Shanghai Municipality (21ZR1473300)

作者简介：赵 伟(1987-), 男, 副研究员. E-mail: zhaowei220@mail.sic.ac.cn

ZHAO Wei (1987-), male, associate professor. E-mail: zhaowei220@mail.sic.ac.cn

通信作者：黄富强，研究员. E-mail: huangfa@mail.sic.ac.cn HUANG Fuqiang, professor. E-mail: huangfq@mail.sic.ac.cn 
金属氰胺化合物 $\mathrm{M}_{x}(\mathrm{NCN})_{y}(\mathrm{M}$ 可为碱金属、碱 土金属、稀土、过渡金属等元素)是衍生自单氰胺 $\mathrm{H}_{2} \mathrm{NCN}$ 的一种化合物。由于阴离子基团 $[\mathrm{NCN}]^{2-}$ 与 $\mathrm{O}^{2-} / \mathrm{S}^{2-}$ 具有等量的形式电荷, 且电负性介于二者之 间，金属氧胺化合物通常具有与其对应的氧化物、 硫化物类似的结构与性质 ${ }^{[1]}$ 。 $[\mathrm{NCN}]^{2-}$ 具有不同的离 子构型，包括对称型的碳二亚胺结构 $[\mathrm{N}=\mathrm{C}=\mathrm{N}]^{2-}$ 和 非对称型的氰胺结构 $[\mathrm{N}-\mathrm{C} \equiv \mathrm{N}]^{2-}$, 二者被认为共振 存在 ${ }^{[2]}$, 它们与金属离子产生不同的配位方式, 使 得金属氧胺化合物具有丰富的晶体结构。另外, $[\mathrm{NCN}]^{2-}$ 的各向异性和碳氮共轭 $\pi$ 键，赋予金属氧胺 化合物独特的物理化学性质。

$\mathrm{M}_{x}(\mathrm{NCN})_{y}$ 的发现可追溯至 20 世纪上半叶, 早 在 1927 年, 就有研究利用 X 射线分析确定了 CaNCN 的结构 ${ }^{[3]}$ 。1970 年代以后, 其他碱金属、碱 土金属氰胺化合物也被成功制备 ${ }^{[4-5]}$ 。受限于其它主 族元素或过渡金属与 $[\mathrm{NCN}]^{2-}$ 的“软硬失配”，传统 合成方法难以制备这些亚稳的化合物。直到本世纪 初, 合成方法的创新才使得这类化合物的制备及结 构表征成为可能 ${ }^{[6-8]}$ 。目前已知的金属氰胺化合物有 八十多种 ${ }^{[9]}$ 。

随着新型合成方法的开发及物化性质的深入研 究, 金属氰胺化合物已在多个领域展现出应用前景, 包括固态发光、光/电催化、电化学储能等 ${ }^{[10-17]}$ 。根 据金属氰胺化合物的结构特点, Eguia-Barrio ${ }^{[18]}$ 和 Sougrati ${ }^{[19]}$ 等率先研究了其作为锂钠离子电池电极 材料的存储性能, 发现其电化学特性比对应氧化物 更优异, 开创了负极材料新体系, 引起了研究者的 高度关注和后续的研究热潮。鉴于此, 本文将介绍 金属氧胺化合物的晶体结构与物化性质, 总结固/液 相合成方法以及金属氰胺化合物在锂钠离子电池领 域应用的代表性研究成果。

\section{1 晶体结构与物化性质}

\section{1 晶体结构}

准线性 $[\mathrm{NCN}]^{2-}$ 阴离子赋予了金属氰胺化合物 空旷和具有孔道的晶体结构, 其典型配位方式如 图 1 所示。其中, 对于 $\mathrm{s}$ 区和 $\mathrm{d}$ 区元素, 最常见的是 八面体六配位, 例如 $\mathrm{MnNCN}$ 具有扭曲岩盐结构 (图 1(a)), $\mathrm{Mn}^{2+}$ 与六个 $[\mathrm{NCN}]^{2-}$ 配位, 立方密排堆积, 取向平行的 $[\mathrm{NCN}]^{2-}$ 构筑了一维孔道结构, 其孔道 尺寸大于 $0.30 \mathrm{~nm}^{[20]}$ 。对于 $\mathrm{ds}$ 区元素，一般为四配 位, 如 $\mathrm{ZnNCN}^{[21]}$ 和 $\mathrm{CuNCN}^{[22]}$ 。 $\mathrm{ZnNCN}$ 结构中, $\mathrm{Zn}^{2+}$ 与四个 $[\mathrm{NCN}]^{2-}$ 配位形成四面体, 孔道尺寸为 $0.315 \mathrm{~nm}$, 而 $\mathrm{CuNCN}$ 中的 $\mathrm{Cu}^{2+}$ 与四个 $[\mathrm{NCN}]^{2-}$ 配位 形成平面四边形, 准层状结构, 层间距大于 $0.30 \mathrm{~nm}$, $\mathrm{HgNCN}^{[23]}$ 则是线性二配位(图 1(b))。 $\mathrm{p}$ 区的 $\mathrm{Sn}^{2+}$ 和 $\mathrm{Pb}^{2+}$ 具有孤对电子, 能以四配位或五配位形成畸变 多面体(图 1(d))。 $\mathrm{SnNCN}^{[24]}$ 的孔道尺寸为 $0.25 \mathrm{~nm}$, $\mathrm{PbNCN}^{[6]}$ 的孔道尺寸为 $0.268 \mathrm{~nm}$, 略小于其它金属 氯胺化合物。

金属氧胺化合物的结构中可存在多种阴、阳离 子, 形成多元化合物, 包括多阳离子化合物, 如 $\mathrm{LiM}(\mathrm{NCN})_{2}\left(\mathrm{M}^{3+} \text { 为 } \mathrm{Al}^{3+} 、 \mathrm{Yb}^{3+} 、 \mathrm{In}^{3+} 、 \mathrm{Ln}^{3+}\right)^{[25]}$ 、 $\mathrm{A}_{2} \mathrm{M}(\mathrm{NCN})_{3}\left(\mathrm{~A} \text { 为 } \mathrm{Li}^{+} 、 \mathrm{Na}^{+}, \mathrm{M}^{4+} \text { 为 } \mathrm{Sn}^{4+} 、 \mathrm{Zr}^{4+} 、 \mathrm{Hf}^{4+}\right)^{[26]}$ 和 $\mathrm{MZn}(\mathrm{NCN})_{2}\left(\mathrm{M}^{2+} \text { 为 } \mathrm{Sr}^{2+} 、 \mathrm{Ba}^{2+}\right)^{[27-28]}$ 等; 多阴离子 化合物，如 $(\mathrm{BiO})_{2} \mathrm{NCN} 、 \mathrm{Sn}_{2} \mathrm{ONCN}$ 和 $(\mathrm{LnO})_{2} \mathrm{NCN}$ $\left(\mathrm{Ln}^{3+} \text { 为 } \mathrm{f} \text { 区稀土离子 }\right)^{\text {等 }}{ }^{[29-32]} 。(\mathrm{LnO})_{2} \mathrm{NCN}$ 的配位方 式如图 1(c) 所示, $\mathrm{Ln}^{3+}$ 为七配位, 每个 $\mathrm{Ln}^{3+}$ 与 4 个 $\mathrm{O}^{2-}$ 和 3 个 $[\mathrm{NCN}]^{2-}$ 配位, $\mathrm{Ln}^{3+}$ 层之间形成的孔道尺寸为 $0.33 \mathrm{~nm}$, 与 $\mathrm{MnNCN}$ 和 $\mathrm{ZnNCN}$ 相比, 更高的配位数 赋予其更大的孔道尺寸。采用以上配位方式形成的 金属氰胺化合物的晶体结构见表 1 。 (a)

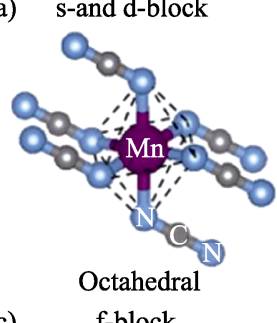

(c)

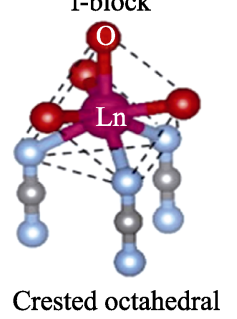

(b)

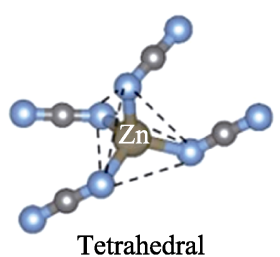

(d) ds-block

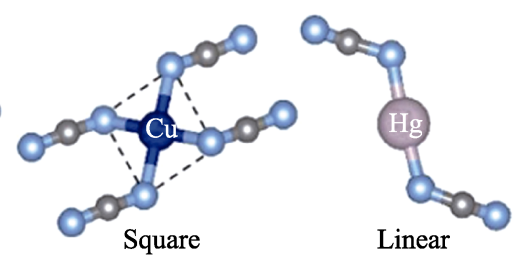

p-block

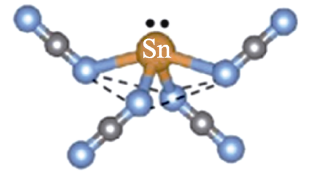

Distorted square-pyramidal

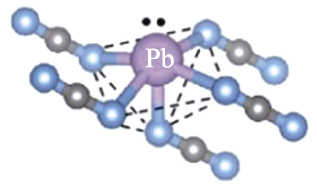

Square-pyramidal

图 1 金属氰胺化合物的典型配位方式 ${ }^{[9]}$

Fig. 1 Typical coordination geometries of metal cyanamides/carbodiimides ${ }^{[9]}$ 
表 1 目前已报道的部分金属氧胺化合物的晶体结构及电化学储能应用 ${ }^{[9]}$

Table 1 Crystal structures of partial metal cyanamides/carbodiimides reported in the literature and their applications for electrochemical energy storage ${ }^{[9]}$

\begin{tabular}{|c|c|c|c|}
\hline Crystal system & Space group & Compounds & Electrochemical energy storage \\
\hline \multirow{4}{*}{ Monoclinic } & $\mathrm{C} 2 / \mathrm{m}$ & $\mathrm{Na}_{2} \mathrm{NCN}, \mathrm{K}_{2} \mathrm{NCN}, \mathrm{Sm}_{2}(\mathrm{NCN})_{3}$ & \multirow[b]{6}{*}{$\begin{array}{l}\text { Anode materials for lithium ion battery: } \\
\mathrm{Ag}_{2} \mathrm{NCN}, \mathrm{SnNCN}, \mathrm{MnNCN}, \mathrm{CuNCN} \text {, } \\
\mathrm{Cr}_{2}(\mathrm{NCN})_{3}, \mathrm{FeNCN}, \mathrm{CoNCN}, \mathrm{NiNCN} \text {, } \\
\mathrm{PbNCN}, \mathrm{Sn}_{2} \mathrm{ONCN} \text {, and } \mathrm{ZnNCN}\end{array}$} \\
\hline & $\mathrm{P} 2_{1} / \mathrm{c}$ & $\mathrm{Ag}_{2} \mathrm{NCN}, \mathrm{HgNCN}$ & \\
\hline & $\mathrm{I} 2 / \mathrm{a}$ & $\mathrm{SnNCN}$ & \\
\hline & $\mathrm{C} 2 / \mathrm{c}$ & $\mathrm{La}_{2} \mathrm{O}(\mathrm{NCN})_{2}$ & \\
\hline \multirow[b]{2}{*}{ Trigonal } & $\mathrm{R}-3 \mathrm{~m}$ & $\mathrm{MgNCN}, \mathrm{CaNCN}, \mathrm{MnNCN}, \mathrm{SrNCN}$ & \\
\hline & $\mathrm{P}-3 \mathrm{~m} 1$ & $\begin{array}{l}\mathrm{In}_{2}(\mathrm{NCN})_{3}, \mathrm{Cr}_{2}(\mathrm{NCN})_{3}, \mathrm{Lu}_{2}(\mathrm{NCN})_{3}, \mathrm{BaNCN} \\
(\mathrm{PrO})_{2} \mathrm{NCN},(\mathrm{NdO})_{2} \mathrm{NCN},(\mathrm{ErO})_{2} \mathrm{NCN},(\mathrm{YO})_{2} \mathrm{NCN} \\
(\mathrm{SmO})_{2} \mathrm{NCN},(\mathrm{EuO})_{2} \mathrm{NCN},(\mathrm{TmO})_{2} \mathrm{NCN},(\mathrm{YbO})_{2} \mathrm{NCN} \\
(\mathrm{DyO})_{2} \mathrm{NCN},(\mathrm{HoO})_{2} \mathrm{NCN}\end{array}$ & \\
\hline Hexagonal & $\mathrm{P} 63 / \mathrm{mmc}$ & FeNCN, CoNCN, NiNCN & \multirow{8}{*}{$\begin{array}{l}\text { Anode materials for sodium ion battery: } \\
\text { FeNCN, CoNCN, NiNCN, MnNCN, } \\
\text { ZnNCN, and CuNCN }\end{array}$} \\
\hline \multirow{4}{*}{ Orthorhombic } & Pbcn & $\mathrm{Zr}(\mathrm{NCN})_{2}, \mathrm{Hf}(\mathrm{NCN})_{2}$ & \\
\hline & Pna21 & $\mathrm{PbNCN}$ & \\
\hline & $\mathrm{Cmcm}$ & $\mathrm{CuNCN}$ & \\
\hline & Pcen & $\mathrm{Sn}_{2} \mathrm{ONCN}$ & \\
\hline \multirow{2}{*}{ Tetragonal } & $\mathrm{I} 4 / \mathrm{mmm}$ & $\mathrm{Li}_{2} \mathrm{NCN},(\mathrm{BiO})_{2} \mathrm{NCN}$ & \\
\hline & $\mathrm{I}-42 \mathrm{~d}$ & $\mathrm{ZnNCN}$ & \\
\hline Triclinic & $\mathrm{P}-1$ & $\mathrm{Tl}_{2} \mathrm{NCN}$ & \\
\hline
\end{tabular}

\section{2 物理化学性质}

$[\mathrm{NCN}]^{2-}$ 阴离子不仅构筑了金属氰胺化合物多 种晶体结构，而且赋予其丰富的物理化学性质。 $[\mathrm{NCN}]^{2-}$ 的电负性小于 $\mathrm{O}^{2-}$, 导致金属氧胺化合物的 价带位置比氧化物更高, 其带隙一般比对应氧化物 小。更小的带隙、空旷的结构和共轭阴离子构型赋 予了金属氧胺化合物较高的电子电导率和离子电导 率, 因此 $\mathrm{FeNCN}, \mathrm{CoNCN}$ 和 $\mathrm{NiNCN}$ 被认为是金属 化合物或半金属化合物 ${ }^{[3]}$ 。大部分金属氰胺化合物对 可见光具有良好的吸收性能。 $\mathrm{Ag}_{2} \mathrm{NCN}^{[12]} 、 \mathrm{CuNCN}^{[22]}$ 是典型的具有光电响应的氰胺化合物。金属氰胺化 合物的磁性与其对应氧化物相似, 以反铁磁性为主, 常见于 $\mathrm{Mn}^{2+}$ 等二价金属离子的化合物 ${ }^{[34]}$ 。 $\mathrm{Cr}_{2}(\mathrm{NCN})_{3}$ 表现出铁磁性 ${ }^{[35]}$, 而其对应的氧化物 $\mathrm{Cr}_{2} \mathrm{O}_{3}$ 呈现反 铁磁性, 可能是更共价的 $\mathrm{Cr}-\mathrm{N}$ 键和更低的配体场 所导致。几乎所有的金属氰胺化合物都能在中性和 碱性溶液中稳定存在，其在酸性环境中的稳定性 较差, $[\mathrm{NCN}]^{2-}$ 倾向于与 $\mathrm{H}^{+}$结合, 进而分解 ${ }^{[36]}$ 。

\section{2 合成方法及形貌调控}

\section{1 固相合成法}

(1)碱金属离子交换法

碱金属离子交换方法以 $\mathrm{s}$ 区碱金属或 $\mathrm{ds}$ 区过渡 金属氰胺化合物 $\left(\mathrm{Li}_{2} \mathrm{NCN} 、 \mathrm{Na}_{2} \mathrm{NCN}\right.$ 和 $\left.\mathrm{ZnNCN}\right)$ 为反 应物，高温下通过与金属卤化物进行阳离子交换获
得其它金属氰胺化合物。Löber 等 ${ }^{[24]}$ 通过 $\mathrm{Li}_{2} \mathrm{NCN}$ 与 $\mathrm{SnCl}_{2} / \mathrm{SnF}_{2}$ 在 $\mathrm{Ar}$ 气氛中反应, 在 $450{ }^{\circ} \mathrm{C}$ 制备出 SnNCN。Srinivasan ${ }^{[37]}$ 和 Neukirch ${ }^{[38]}$ 等通过 $\mathrm{LnCl}_{3}$ $\left(\mathrm{Ln}=\mathrm{La}, \mathrm{Ce}, \mathrm{Pr}\right.$ ) 和 $\mathrm{Li}_{2} \mathrm{NCN}$ 的混合物在 $600{ }^{\circ} \mathrm{C}$ 下反应 制备了系列多阴离子稀土氰胺化合物 $\mathrm{Ln}(\mathrm{NCN}) \mathrm{Cl}$ 。 Dronskowski 等 ${ }^{[39]}$ 将 $\mathrm{PbCl}_{2}$ 和 $\mathrm{Na}_{2} \mathrm{NCN}$ 按摩尔比反 应得到单相多晶 $\mathrm{PbNCN}$ ，反应方程式为:

$$
\mathrm{Na}_{2} \mathrm{NCN}+\mathrm{PbCl}_{2}=\mathrm{PbNCN}+2 \mathrm{NaCl}
$$

其中, $\mathrm{Na}^{+}$是一种常见的 “硬酸”, 而 $[\mathrm{NCN}]^{2-}$ 尺寸大、 电子云易变形, 相比 $\mathrm{Cl}^{-}$更软。根据软硬酸碱理论 “软亲软, 硬亲硬”, $\mathrm{NaCl}$ 比 $\mathrm{Na}_{2} \mathrm{NCN}$ 更稳定, 可以 促进反应产生 $\mathrm{PbNCN}$ 。将 $\mathrm{BiOCl}$ 和 $\mathrm{Na}_{2} \mathrm{NCN}$ 按摩 尔比反应获得了 $\mathrm{Bi}_{2} \mathrm{O}_{2} \mathrm{NCN}$, 其晶体结构与 $\mathrm{Bi}_{2} \mathrm{O}_{2} \mathrm{X}$ $(\mathrm{X}=\mathrm{S}, \mathrm{Se}, \mathrm{Te})$ 相似 ${ }^{[29]}$ 。

\section{(2)三聚氧胺热解法}

碱金属离子交换法操作简单、普适性强，在新 型金属氰胺化合物的合成上获得了广泛应用。然而， 其合成温度往往较高。这是因为 $[\mathrm{NCN}]^{2-}$ 来源于反应 物中的离子键断裂, 该过程需要较多能量。此外, 该 方法获得的产物粒径较大、形貌不规整，尺寸一般 在微米级别。因此, 迫切需要一种合适的 $[\mathrm{NCN}]^{2-}$ 源, 降低合成温度，控制产物尺寸及形貌。

单氧胺 $\mathrm{H}_{2} \mathrm{NCN}$ 及其衍生的双氰胺和三聚氭胺 也可作为 $[\mathrm{NCN}]^{2-}$ 源。根据 Thomas 等 ${ }^{[40]}$ 报道的三聚 氧胺高温热解过程, 其在 $350{ }^{\circ} \mathrm{C}$ 时重排得到三嗪, 作为中间体可以降低活化能，促进 $\mathrm{C}-\mathrm{N}$ 断裂并形成 $[\mathrm{NCN}]^{2-}$ 。碱土金属氧胺化合物 $\mathrm{MgNCN} 、 \mathrm{SrNCN}$ 和 
$\mathrm{BaNCN}$ 可通过三聚氰胺与金属氮化物 $\left(\mathrm{Mg}_{3} \mathrm{~N}_{2}, \mathrm{Sr}_{2} \mathrm{~N}\right.$, $\mathrm{Ba}_{3} \mathrm{~N}_{2}$ ) 在 $740{ }^{\circ} \mathrm{C}$ 至 $850{ }^{\circ} \mathrm{C}$ 衫烧制备 ${ }^{[4]}$ 。Cao 课题组 ${ }^{[16]}$ 将辛酸、三聚氰胺和氯化锰经水热反应获得的产物 置于惰性气氛中 $500{ }^{\circ} \mathrm{C}$ 烠烧得到片状 $\mathrm{MnNCN}$ 。Yan 等 ${ }^{[41]}$ 通过熔融盐热解亚铁三聚氰胺配合物制备 FeNCN 纳米棒, 熔融盐反应环境对 FeNCN 的取向 生长至关重要, 在无熔融盐条件下只能获得 FeNCN 纳米颗粒。

相比碱金属离子交换法, 三聚氧胺热解法的合 成温度相对较低。温和的合成条件使得产物形貌、 尺寸更加可控。高温热解后, 生成的金属氰胺化合 物一般可以保持前驱体的形貌(例如片状、棒状等)。 目前, 金属氧胺化合物的形貌调控仍处于初级阶段, 需要进一步优化固相合成工艺控制合成产物尺寸和 微结构等。

\section{(3)中间体热解法}

$\mathrm{d}$ 区过渡金属离子(如 $\mathrm{Fe}^{2+} 、 \mathrm{Co}^{2+} 、 \mathrm{Ni}^{2+}$ 等)的电 子云变形困难, 易捕获电子而形成 $3 \mathrm{~d}^{10}$ 满壳层结构, 较 $\mathrm{ds}$ 区的 $\mathrm{Zn}^{2+}$ 、 $\mathrm{Ag}^{+}$等更“硬”, 与 $\mathrm{NCN}^{2-}$ 的键合力 不强, 很难与质子完全交换。其在水溶液中反应时, 先形成 $\mathrm{M}(\mathrm{HNCN})_{2}$ 中间体, 再通过高温脱氢获得对 应的氰胺化合物 ${ }^{[2-44]}$ 。

\section{2 液相合成法}

固相合成法操作简单，但条件苛刻、反应缓慢、 形貌及尺寸控制困难。基于液相反应的制备策略克 服了固相合成的缺点, 也常用于合成金属氰胺化合 物。常见的湿化学合成法包括共沉淀、溶胶一凝胶、 溶剂热、微乳液、自组装等。

以 $\mathrm{H}_{2} \mathrm{NCN}$ 溶液为反应介质制备金属氰胺化合 物已有大量报道, 在溶液环境中 $\mathrm{H}_{2} \mathrm{NCN}$ 脱去质子 形成 $[\mathrm{NCN}]^{2-}$ 源，与金属离子反应后获得对应氰胺 化合物, 在水溶液及有机体系均能进行反应。

\section{(1)水系共沉淀}

一些金属阳离子可与 $\mathrm{H}_{2} \mathrm{NCN}$ 水溶液直接反应 得到对应氰胺化合物, 如 $\mathrm{Zn}^{2+} 、 \mathrm{Cu}^{2+} 、 \mathrm{Ag}^{+} 、 \mathrm{Cd}^{2+}$ 和 $\mathrm{Tl}^{+}$等 $[12,21-23,45]$ 。部分金属阳离子不能直接取代 $\mathrm{H}^{+}$, 需借助碱来捕获质子以释放 $[\mathrm{NCN}]^{2-}, \mathrm{HgNCN}$ 、 $\mathrm{Tl}_{2} \mathrm{NCN}^{[46]}$ 等可在碱性水溶液中生成。但是, $[\mathrm{NCN}]^{2-}$ 在结合金属阳离子 (如 $\mathrm{Fe}^{2+} 、 \mathrm{Cu}^{2+}$ ) 时伴随着 $\mathrm{OH}^{-}$的竞 争。 $\mathrm{NH}_{3}$ 含有孤对电子, 不仅能够结合 $\mathrm{H}^{+}$以释放 $[\mathrm{NCN}]^{2-}$, 而且能络合金属阳离子, 有效抑制高 $\mathrm{pH}$ 时氢氧化物的生成。黄课题组 ${ }^{[12]}$ 率先报道了 $\mathrm{NH}_{3}$ 浓度在金属氰胺化合物形貌演化中的关键作用, 高 比例的 $\left[\mathrm{NH}_{3}\right] /\left[\mathrm{M}^{z+}\right]$ 有利于大晶粒尺寸, 而在较低比 例下容易得到由纳米颗粒组成的二级颗粒。反应机
理表明, $\mathrm{NH}_{3}$ 络合金属阳离子可以调控成核-生长过 程, 高比例 $\left[\mathrm{NH}_{3}\right] /\left[\mathrm{M}^{z+}\right]$ 会导致成核减少并促进晶粒 长大。因此, 调整 $\left[\mathrm{NH}_{3}\right] /\left[\mathrm{M}^{z+}\right]$ 比例可以制备不同尺 寸及形貌的金属氰胺化合物。

如上所述，水系共沉淀制备金属氰胺化合物需 满足以下两个条件: (1) $\mathrm{H}_{2} \mathrm{NCN}$ 解离释放 $[\mathrm{NCN}]^{2-}$; (2)阳离子与 $[\mathrm{NCN}]^{2-}$ 络合前保持稳定, 避免与 $\mathrm{OH}^{-}$ 等沉淀形成副产物。利用合适的碱可促进晶体成核、 生长, 实现液相可控合成。其中, $\mathrm{NH}_{3}$ 是一种高效的 无机碱, 起到促进 $[\mathrm{NCN}]^{2-}$ 释放、保护阳离子、调控 成核一生长过程等多重作用。

\section{(2)有机系共沉淀}

水系共沉淀存在无机碱种类圆乏、副产物多、 反应过程调控困难等缺点, 使得金属氰胺化合物的 可控合成受到很大限制。有机碱因其种类丰富、无 氢氧化物副产物、可利用的表面活性剂的配体效应 等优势，逐渐被用于辅助合成金属氰胺化合物，尤 其是用于获得纳米尺寸的产物。

质子是一种“硬酸”, 理论上, 可以选用比 $[\mathrm{NCN}]^{2-}$ 更“硬”的有机路易斯碱来促进 $\mathrm{H}_{2} \mathrm{NCN}$ 的解 离。有机胺 $\left(\mathrm{R}-\mathrm{NH}_{\delta}, \delta=0,1,2\right)$ 是一类常用的有机碱 和表面活性剂，烷基“R-”的丰富多样化赋予其“硬 度”可调性及形貌调控等诸多优点。

目前，已报道的用于合成金属氰胺化合物的有 机胺体系包括油胺、十六烷胺、十八烷胺和茮胺 等 ${ }^{[36,47-49]}$ 。黄课题组 ${ }^{[48]}$ 开发了一种在有机体系中合 成金属氰胺化合物的通用策略，以甲苯/乙醇溶剂为 反应介质, 油胺、十六烷胺、十八烷胺等伯胺作为 表面活性剂, 实现了金属氰胺化合物的尺寸和形貌 可控合成, 制备出 $\mathrm{Ag}_{2} \mathrm{NCN}$ 纳米棒、 $\mathrm{ZnNCN}$ 纳米棒 和 $\mathrm{PbNCN}$ 纳米颗粒。制备的 $\mathrm{Ag}_{2} \mathrm{NCN}$ 纳米棒具有 更好的结晶性和更高的比表面积, 与在水溶液中沉 淀得到的 $\mathrm{Ag}_{2} \mathrm{NCN}$ 微米晶相比, 其光催化性能显著 增强。之后, 黄课题组 ${ }^{[36,47]}$ 通过无水乙醇溶解氰胺 与金属卤化物, 形成均相反应体系, 加入有机强碱 茮胺, 调控反应过程, 制备了尺寸小于 $10 \mathrm{~nm}$ 且单 分散的 CdNCN、MnNCN 纳米颗粒。Koziej 等 ${ }^{[49]}$ 也报道了茮胺在合成金属氰胺化合物的作用。他们 将金属卤化物分散到熔融 $\mathrm{H}_{2} \mathrm{NCN}$ 中, 加入茮胺促 进 $\mathrm{H}_{2} \mathrm{NCN}$ 解离和快速反应成核, 制备出尺寸小于 $30 \mathrm{~nm}$ 的 $\mathrm{CuNCN} 、 \mathrm{MnNCN}$ 纳米颗粒。然而, 在这 一制备方法中, 熔融 $\mathrm{H}_{2} \mathrm{NCN}$ 和金属卤化物之间的 剧烈非均相反应过程导致明显的颗粒团聚。

到目前为止，通过液相法合成金属氰胺化合物 的报道越来越多。尽管形貌、尺寸调控还处于初级 
阶段, 但是液相法在金属氰胺化合物合成领域已显 示出较大的潜力, 尤其是合成纳米尺度的氰胺化合 物展现了独特的优势。图 2 为部分已合成的金属氰 胺化合物的 $\operatorname{SEM}$ (前三排)、TEM(第四排)照片。

\section{3 电化学储能应用}

金属氯胺化合物具有独特的晶体结构，兼具较 高的电子电导率和离子电导率, 在发光、储能、光/ 电催化等领域具有潜在的应用前景。2016 年, EguiaBarrio $^{[18]}$ 和 Sougrati ${ }^{[19]}$ 等分别独立报道了金属氰胺 化合物在电化学储能领域的应用, 主要研究了 $\mathrm{MNCN}(\mathrm{M}=\mathrm{Cu} 、 \mathrm{Zn} 、 \mathrm{Mn} 、 \mathrm{Fe} 、 \mathrm{Co}$ 和 $\mathrm{Ni})$ 作为锂钠 离子电池电极材料的电化学性能, 开启了一类新型

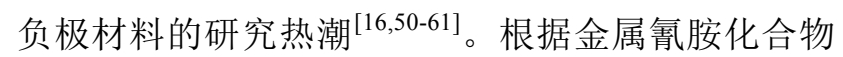
的晶体结构、电子结构特点, 其作为锂钠离子电池 负极材料, 和对应氧化物相比具有以下优点: (1) $[\mathrm{NCN}]^{2-}$ 与 $\mathrm{O}^{2-}$ 相比电负性较小, 氧化还原反应所需 能量更少, 电化学反应平台更低; $(2)[\mathrm{NCN}]^{2-}$ 的共轭
结构及多原子轨道杂化促进电子离域, 有利于电子 导电, 提高倍率性能; (3) $[\mathrm{NCN}]^{2-}$ 的线性结构使得化 合物晶体结构空旷, 充放电过程中可以有效缓解体 积膨胀，增强循环稳定性。以下内容将结合相关重 要工作介绍金属氰胺化合物作为锂钠离子电池负极 材料的研究进展。

\section{1 锂离子电池}

目前已报道的金属氰胺化合物的储 $\operatorname{Li}($ 或 $\mathrm{Na}$ )的 机制以转化反应为主, 部分包含嵌入反应及合金化 反应。转化反应是负极储锂机制的一种, 常见于过 渡金属氧化物、硫化物、氮化物和磷化物等 ${ }^{[15]}$, 嵌 入反应常见于层状材料, 如石墨、二硫化钼等, 而 $\mathrm{Si} 、 \mathrm{Ge} 、 \mathrm{Sn} 、 \mathrm{Sb}$ 等金属单质会历经合金化反应。金 属氰胺化合物的转化反应方程式如下:

$$
\mathrm{M}_{x}(\mathrm{NCN})_{y}+2 y \mathrm{Li} \Leftrightarrow y \mathrm{Li}_{2} \mathrm{NCN}+x \mathrm{M}
$$

对于 $\mathrm{d}$ 区过渡金属氰胺化合物，一般仅发生转 化反应; 而对于 $\mathrm{ds}$ 区过渡金属氰胺化物(如 $\mathrm{ZnNCN}$ ), 在转化反应之后，还会历经合金化反应，其反应方 程式如下所示:
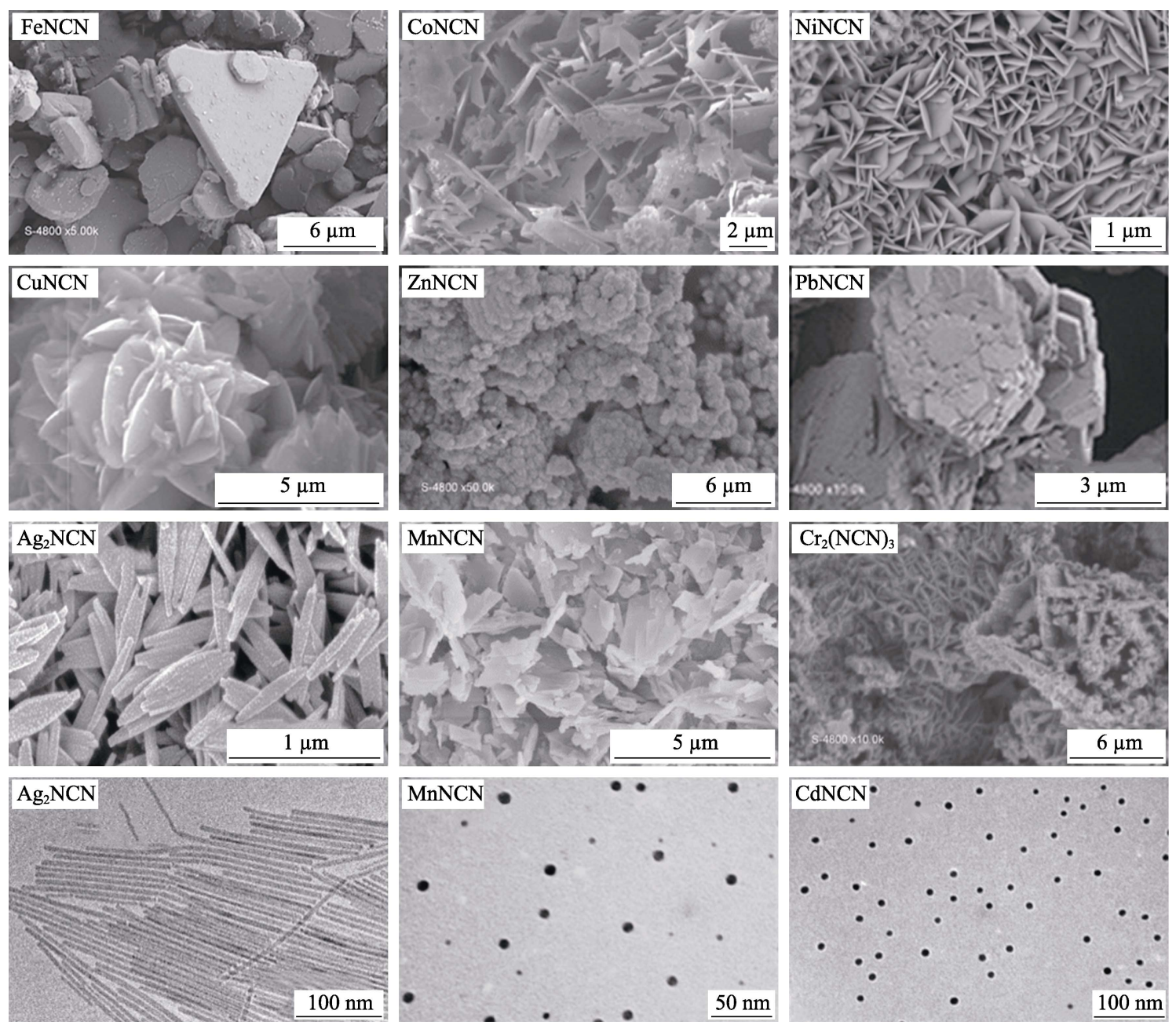

图 2 部分已合成的金属氧胺化合物的 SEM(前三排)、TEM(第四排)照片 ${ }^{[12,16,18,36,47,48,50,52,53]}$

Fig. 2 SEM (the first three rows), TEM (the fourth row) images of partial metal cyanamides/carbodiimides ${ }^{[12,16,18,36,47,48,50,52,53]}$ 


$$
\mathrm{M}+m \mathrm{Li} \Leftrightarrow \mathrm{Li}_{m} \mathrm{M}
$$

一般来说, 转化反应机制可提供较高的比容量。相 较于石墨这种插层型负极, 金属氰胺化合物展现出较 高的理论容量和实际容量。下面将介绍 FeNCN、 $\mathrm{CoNCN} 、 \mathrm{MnNCN} 、 \mathrm{Cr}_{2}(\mathrm{NCN})_{3}$ 和 $\mathrm{Sn}_{2} \mathrm{ONCN}$ 等典型 金属氧胺化合物的储锂性能和电化学反应机制。

FeNCN 在 $0.01 \sim 2.5 \mathrm{~V}$ 的电化学窗口内具有和 $\mathrm{FeO}$ 类似的首圈放电曲线, $0.6 \sim 0.8 \mathrm{~V}$ 左右有平台。 充电后，首圈具有显著的不可逆容量，库仑效率约 为 $70 \%$, 类似于氧化物, 不可逆部分可归因于电解 质分解形成的 SEI 膜 ${ }^{[19]}$ 。接下来的充放电曲线虽然 明显不同于首圈，但完全可逆，库仑效率接近 $97 \%$ 。 另外一个和 $\mathrm{FeO}$ 类似的特点是, $\mathrm{FeNCN}$ 的储锂过程 为单相反应, 放电曲线呈现斜坡, 平均电压约为 $1.4 \mathrm{~V}$ 。微米尺寸的 FeNCN 在 $C / 5$ 倍率下的可逆容 量达 $600 \mathrm{mAh} / \mathrm{g}$, 在 $30 \mathrm{C}$ 高倍率下仍能稳定工作 (图 3(a))。其 $C / 5$ 倍率的循环稳定性远优于 $\mathrm{FeO}$ 及 其复合材料, $\mathrm{FeO} / \mathrm{Li}$ 循环 25 圈后容量降低 $60 \%$, 而 FeNCN/Li 循环 50 圈后降低 20\%, 50 100 圈仅降低 $5 \%$ (图 3(b))。利用原位 XRD、原位 Mössbauer 谱 (图 3(c)) 及非原位 FT-IR 谱研究 FeNCN 的储锂机制, 发现首圈放电后, FeNCN 粉末化并生成 Fe 纳米颗粒 及大量非晶产物, 放电后 $\mathrm{Fe}$ 纳米颗粒再次氧化, 但
不完全, 经历的电化学反应如下:

$$
\mathrm{FeNCN}+2 \mathrm{Li} \Leftrightarrow \mathrm{Fe}+\mathrm{Li}_{2} \mathrm{NCN}
$$

Arayamparambil 等 ${ }^{[51]}$ 深入研究了 CoNCN 储锂 性能和反应机制，与 FeNCN 类似, CoNCN 在 $0.9 \mathrm{~V}$ 左右有平台(图 3(d)), 首圈的不可逆容量较大, 但后 续库仑效率更优, 30 圈后超过 $99.6 \%$ 。CoNCN 也具 有优异的倍率性能和循环稳定性, $4 C$ 下和石墨负极 相当, 比容量达 $360 \mathrm{mAh} / \mathrm{g}, 2 C$ 循环 400 次后仍保持 $530 \mathrm{mAh} / \mathrm{g}$ 左右, 和理论值接近且类似于 $\mathrm{CoO}$ 的性 能(图 3(e))。原位 XRD 和原位 X 射线吸收近边结构 谱(XANES)表明, CoNCN 与 $\mathrm{FeNCN}$ 类似, 历经转换 反应，首圈放电后生成 $\mathrm{Co}$ 纳米颗粒。对比分析本征 CoNCN 和充电产物的 XANES 谱(图 3(f)), 发现 Co 纳米颗粒氧化后的产物具有明显不同于 $\mathrm{CoNCN}$ 的 $\mathrm{Co}-\mathrm{N}$ 配位方式及 $\mathrm{Co}-\mathrm{N}$ 距离, 即 $[\mathrm{NCN}]^{2-}$ 基团的构 型可能在充放电过程之后发生了改变。CoNCN 可能 的储锂机制如下:

$$
\begin{gathered}
\mathrm{CoNCN}+2 \mathrm{Li} \rightarrow \mathrm{Co}+\mathrm{Li}_{2} \mathrm{NCN} \text { (首圈) } \\
\mathrm{Co}+\mathrm{Li}_{2} \mathrm{NCN} \Leftrightarrow \text { “CoNCN”+2Li (循环) }
\end{gathered}
$$

$\mathrm{CoNCN}$ 放电后形成 $\mathrm{Co}$ 纳米颗粒和 $\mathrm{Li}_{2} \mathrm{NCN}$, 充电 后虽然形成“CoNCN”，但 $[\mathrm{NCN}]^{2-}$ 基团的构型极有 可能由对称型 $[\mathrm{N}=\mathrm{C}=\mathrm{N}]^{2-}$ 部分转变为非对称型 $[\mathrm{N}-\mathrm{C} \equiv \mathrm{N}]^{2-}($ 图 3(g))。
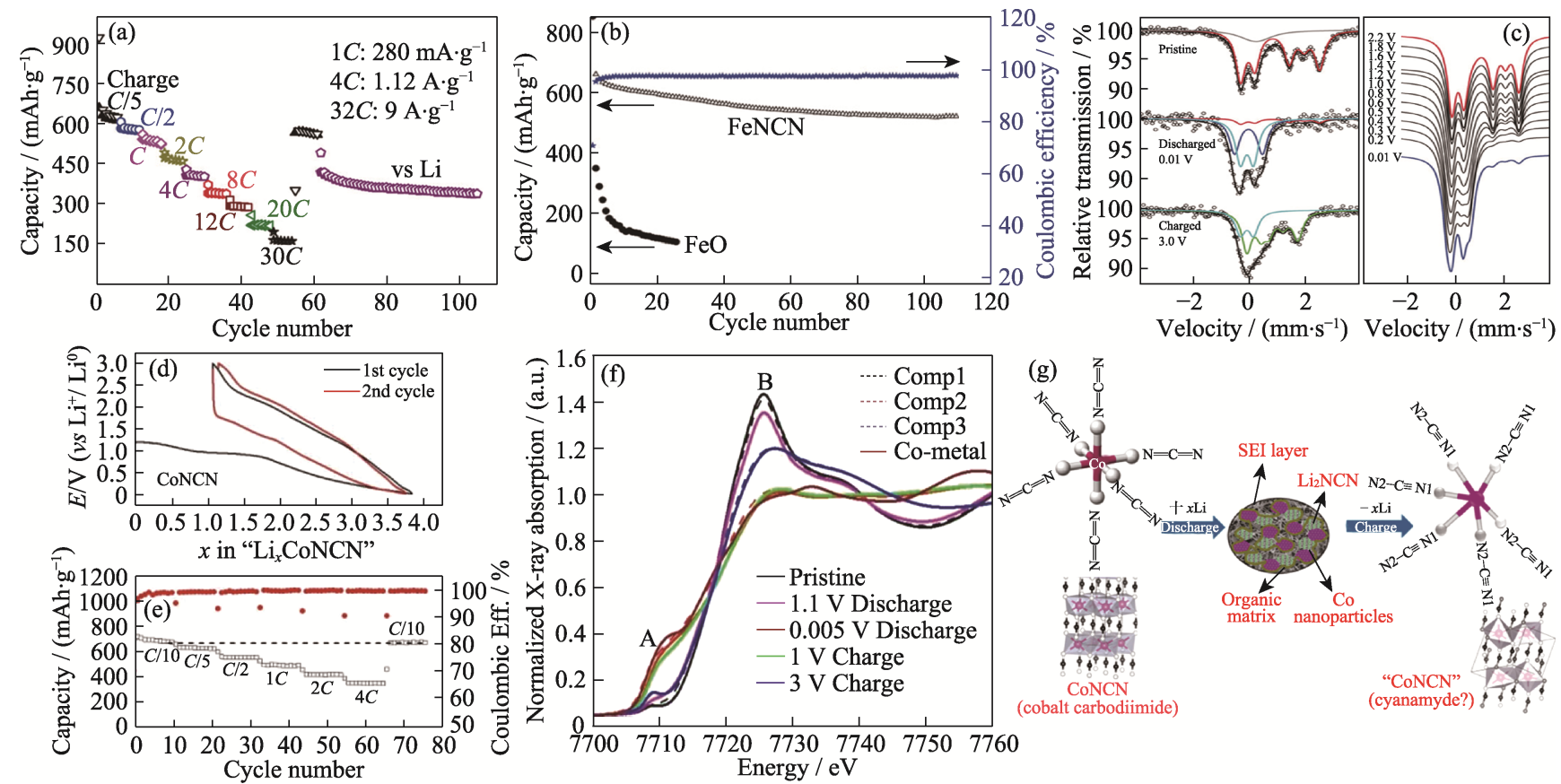

图 $3 \mathrm{FeNCN}$ 和 $\mathrm{CoNCN}$ 用作锂离子电池负极材料的电化学性能和反应机制 ${ }^{[19,51]}$

Fig. 3 Electrochemical performance and charge storage mechanism of FeNCN and CoNCN anode materials for lithium-ion batteries ${ }^{[19,51]}$

(a) Rate capacity of FeNCN; (b) Comparison of specific capacity and Coulombic efficiency between FeNCN and FeO; (c) Mössbauer spectra of FeNCN electrode at different charge and discharge potentials; (d) $1^{\text {st }}$ and $2^{\text {nd }}$ galvanostatic voltage profiles of CoNCN electrode at $\mathrm{C} / 10$ rate; (e) Rate capacity of CoNCN; (f) Co K-edge XANES spectra of CoNCN electrode at different charge and discharge potentials; (g) Schematic illustration of the proposed charge storage mechanism of CoNCN; Colourful figures are available on website 
除二价金属以外, Arayamparambil 等 ${ }^{[53]}$ 研究了 三价金属的氰胺化合物如 $\mathrm{Cr}_{2}(\mathrm{NCN})_{3}$ 的储锂性能。 $\mathrm{Cr}_{2}(\mathrm{NCN})_{3}$ 脱嵌锂过程中, 首圈的不可逆容量较大, 循环 10 圈后，比容量趋于稳定，500 圈后库仑效率 达 99.7\%, 4C 倍率下比容量为 $540 \mathrm{mAh} / \mathrm{g}$, 优于 FeNCN 和 CoNCN(图 4(a,b))。基于原位 XRD 表征 的晶格参数变化(图 4(c,d)) 和非原位 XANES 谱的拟 合参数(图 4(e, f)) 可见, $\mathrm{Cr}_{2}(\mathrm{NCN})_{3}$ 的脱嵌锂过程比 FeNCN、CoNCN 复杂, 充电时 $\mathrm{Cr}_{2}(\mathrm{NCN})_{3}$ 先通过 $\mathrm{Cr}^{3+} \rightarrow \mathrm{Cr}^{2+}$ 路径嵌锂, 再通过 $\mathrm{Cr}^{2+} \rightarrow \mathrm{Cr}^{0}$ 路径发生转 换反应。首次放电后产物为 $\mathrm{Li}_{x} \mathrm{Cr}_{2}(\mathrm{NCN})_{3} 、 \mathrm{Li}_{2} \mathrm{NCN}$ 和 $\mathrm{Cr}$ 纳米颗粒的混合物; 充电后, 大部分 $\mathrm{Cr}$ 金属再
氧化成 $\mathrm{Cr}^{3+}$, 但包含少量 $\mathrm{Cr}^{2+}$ 。这一过程与 $\mathrm{Cr}-\mathrm{Li}_{2} \mathrm{NCN}-\mathrm{Cr}_{2}(\mathrm{NCN})_{3}$ 三元相图、产物形成能及嵌 锂曲线的理论模拟结果相一致(图 $4(\mathrm{~g} \sim \mathrm{i}))_{0}$ 。 $\mathrm{Cr}_{2}(\mathrm{NCN})_{3}$ 可能的储锂机制如下:

嵌锂过程

$$
\begin{gathered}
\mathrm{Cr}_{2}(\mathrm{NCN})_{3}+x \mathrm{Li} \rightarrow \mathrm{Li}_{x} \mathrm{Cr}_{2}(\mathrm{NCN})_{3}(x<2) \\
\mathrm{Li}_{x} \mathrm{Cr}_{2}(\mathrm{NCN})_{3}+(6-x) \mathrm{Li} \rightarrow 2 \mathrm{Cr}+3 \mathrm{Li}_{2} \mathrm{NCN}
\end{gathered}
$$

脱锂过程

$$
\begin{aligned}
& 2 \mathrm{Cr}+ 3 \mathrm{Li}_{2} \mathrm{NCN} \rightarrow x \mathrm{Cr}_{2}(\mathrm{NCN})_{3}+ \\
&(1-x) \mathrm{Li}_{2} \mathrm{Cr}_{2}(\mathrm{NCN})_{3}+(6-2 x) \mathrm{Li}
\end{aligned}
$$

$\mathrm{Cr}_{2}(\mathrm{NCN})_{3}$ 储锂包含嵌入脱出和转换反应的混 合过程, 融合了 $\mathrm{Cr}^{3+} \rightarrow \mathrm{Cr}^{2+} \rightarrow \mathrm{Cr}^{0}$ 和 $\mathrm{Cr}^{2+} \rightarrow \mathrm{Cr}^{0}$ 反应路
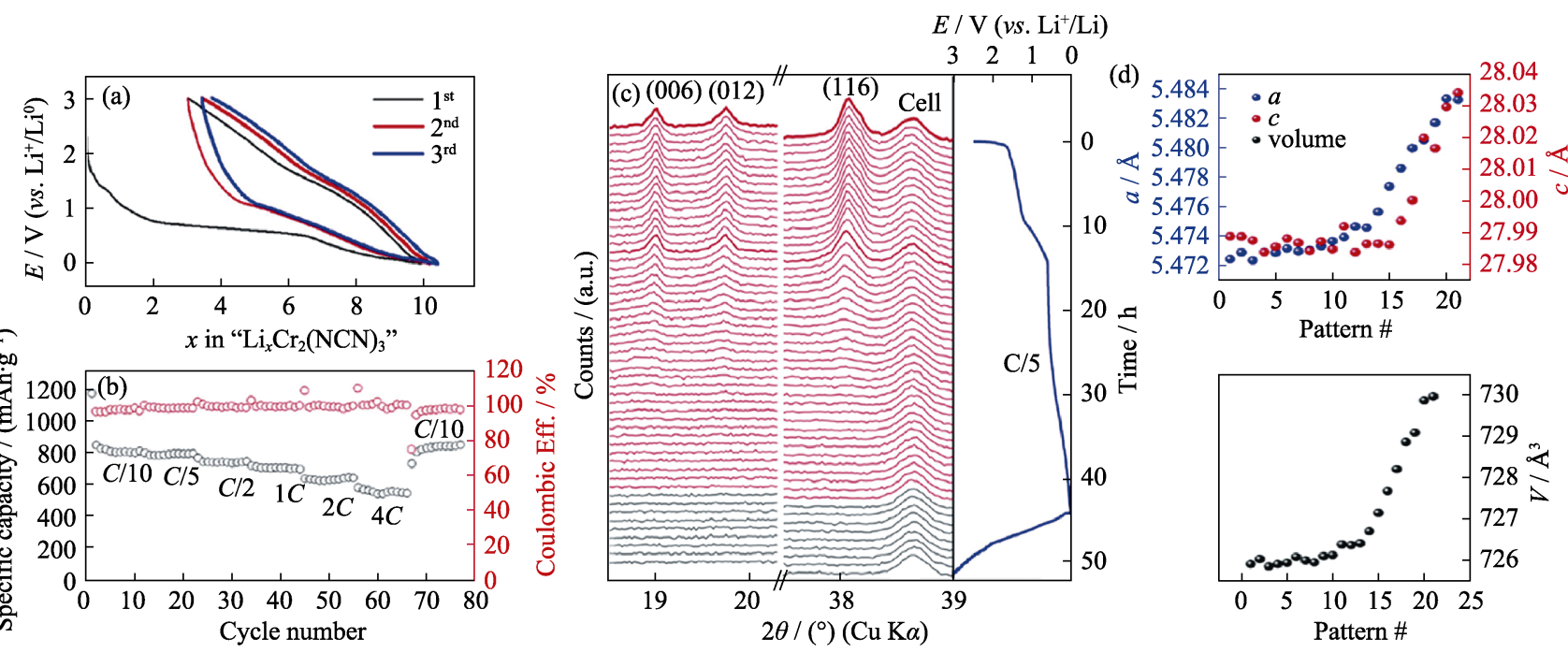
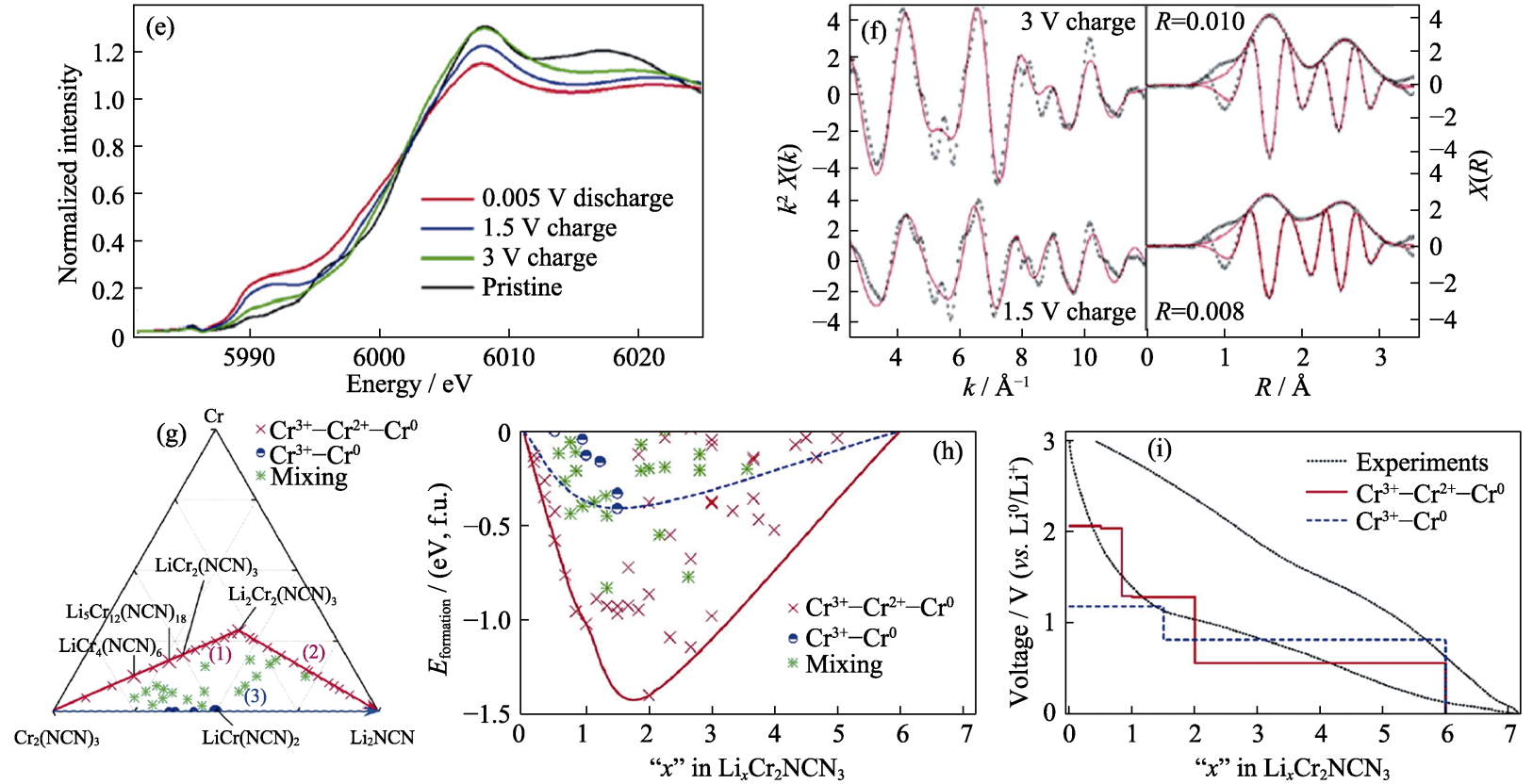

图 $4 \mathrm{Cr}_{2}(\mathrm{NCN})_{3}$ 用作锂离子电池负极材料的电化学性能和反应机制 ${ }^{[53]}$

Fig. 4 Electrochemical performance and charge storage mechanism of $\mathrm{Cr}_{2}(\mathrm{NCN})_{3}$ anode material for lithium-ion battery ${ }^{[53]}$ (a) $1^{\text {st }}, 2^{\text {nd }}$ and $3^{\text {rd }}$ galvanostatic voltage profiles at $C / 10$ rate; (b) Rate capacity; (c) Operando XRD patterns at $C / 5$ rate; (d) Evolution of the corresponding cell parameters and volume; (e) XANES spectra at different charge and discharge potentials; (f) Fit curves of the EXAFS spectra at

1.5 and $3 \mathrm{~V}$ charged states in k- and R-space; (g) Phase diagram for $\mathrm{Cr}-\mathrm{Li}_{2} \mathrm{NCN}-\mathrm{Cr}_{2}(\mathrm{NCN})_{3}$ ternaries; (h) Formation energies of the calculated intermediate compounds; (i) Calculated voltage profile of $\mathrm{Cr}_{2}(\mathrm{NCN})_{3}$ upon lithiation 
径, $\mathrm{Cr}^{3+} / \mathrm{Cr}^{2+} /$ 和 $\mathrm{Cr}^{2+} / \mathrm{Cr}^{0}$ 氧化还原电对都对容量有贡 献, 因此电化学性能更加优异。

以 $\mathrm{SnO}_{2}$ 为代表的锡氧化物的储锂性能已得到 广泛研究, 但是其受限于导电性差和嵌锂前后体积 膨胀导致的粉末化, 循环稳定性不佳 ${ }^{[62]}$ 。Lü 等 ${ }^{[61]}$ 首次研究了锡的氯胺化合物的储锂性能。以 $\mathrm{Sn}_{2} \mathrm{ONCN}$ 为例, 其储锂过程包括类似于 $\mathrm{Fe} 、 \mathrm{Co}$ 和 $\mathrm{Cr}$ 等氰胺化合物历经的转换反应以及 $\mathrm{Sn}$ 的合金反 应，即类似于 $\mathrm{SnO}_{2}$ 负极材料的电化学反应机制:

$\mathrm{Sn}_{2} \mathrm{ONCN}+4 \mathrm{Li} \Leftrightarrow 2 \mathrm{Sn}+\mathrm{Li}_{2} \mathrm{O}+\mathrm{Li}_{2} \mathrm{NCN}$

$\mathrm{Sn}+x \mathrm{Li} \Leftrightarrow \operatorname{Li}_{x} \mathrm{Sn}(0 \leqslant x \leqslant 4.4)$

$\mathrm{Sn}_{2} \mathrm{ONCN}$ 的首圈库仑效率、倍率性能和循环稳 定性均优于常规的 $\mathrm{SnO}_{2}$ 负极材料。电流密度为
$0.5 \mathrm{~A} / \mathrm{g}$ 时, $\mathrm{Sn}_{2} \mathrm{ONCN}$ 的首圈放电、充电容量分别为 1355 和 $1079 \mathrm{mAh} / \mathrm{g}$, 库仑效率约为 $80 \%$, 远高于 $\mathrm{SnO}_{2}$ 的 $61 \%$, 可推测 $\mathrm{Sn}_{2} \mathrm{ONCN}$ 转换反应过程的可 逆性更高。循环 200 圈后 $\mathrm{SnO}_{2}$ 电极衰减至 $100 \mathrm{mAh} / \mathrm{g}$ 以下，而 $\mathrm{Sn}_{2} \mathrm{ONCN}$ 依然保持 $610 \mathrm{mAh} / \mathrm{g}$ 的比容量, 循环稳定性更优。另外, 在电流密度高达 $5 \mathrm{~A} / \mathrm{g}$ 时, $\mathrm{Sn}_{2} \mathrm{ONCN}$ 的比容量为 $455 \mathrm{mAh} / \mathrm{g}$, 而 $\mathrm{SnO}_{2}$ 的比容量几乎可以忽略不计, 证明 $\mathrm{Sn}_{2} \mathrm{ONCN}$ 具有 优异的倍率性能(图 5(a))。添加石墨片 (Graphene nanosheets, GN)构成复合电极 $\mathrm{Sn}_{2} \mathrm{ONCN} / \mathrm{GN}$ 后, 比 容量和倍率性能进一步提升, 电流密度为 $0.1 、 0.2$ 、 $0.5 、 1.0 、 2.0$ 和 $4.0 \mathrm{~A} / \mathrm{g}$ 时，比容量分别为 $1064 、 1015$ 、 964、915、828 和 $735 \mathrm{mAh} / \mathrm{g}$ (图 5(b)), 且在 1.0 和
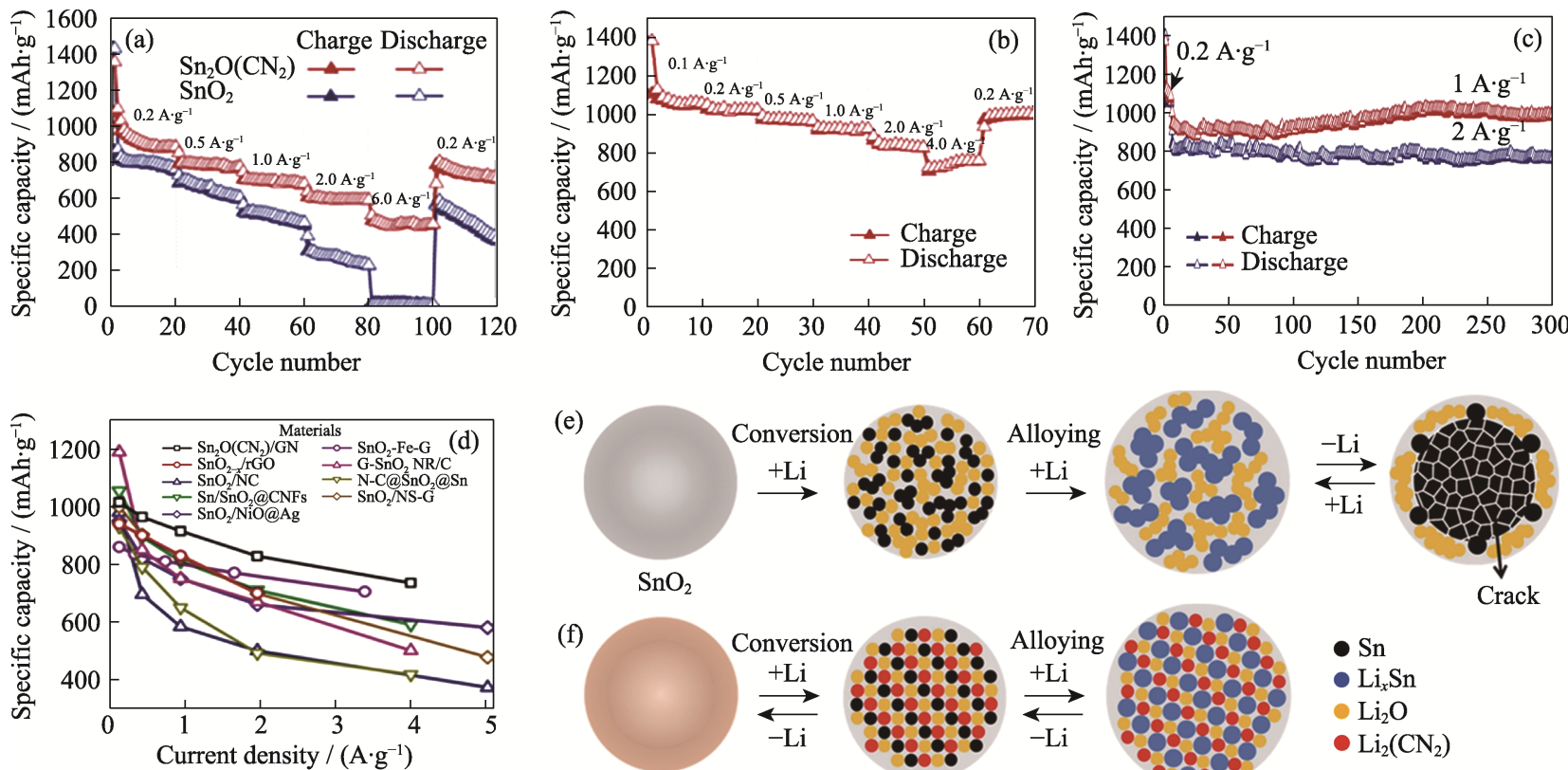

(e)
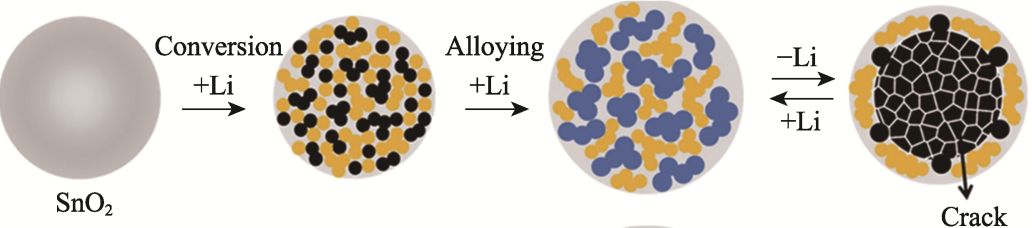

(f)

$\mathrm{SnO}_{2}$
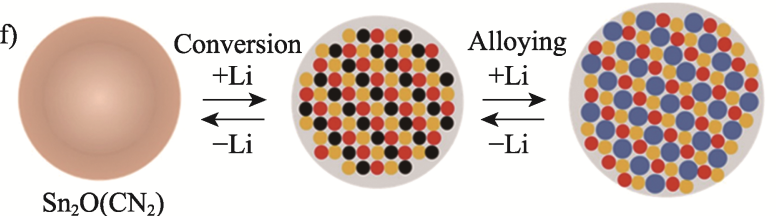

- $\mathrm{Sn}$

- $\mathrm{Li}_{x} \mathrm{Sn}$

- $\mathrm{Li}_{2} \mathrm{O}$

- $\mathrm{Li}_{2}\left(\mathrm{CN}_{2}\right)$

(g)

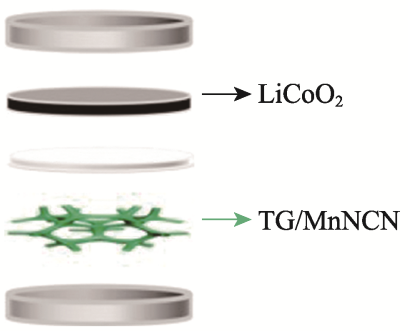

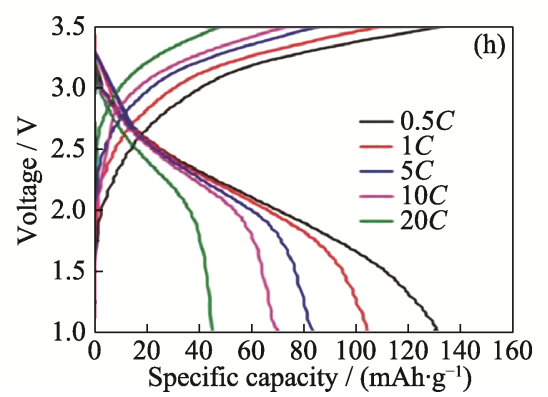

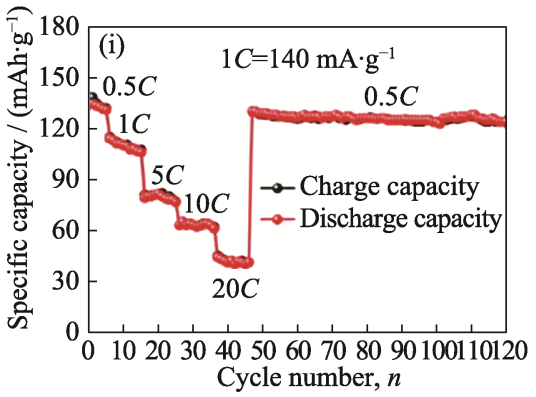

图 5 (a) $\mathrm{Sn}_{2} \mathrm{ONCN}$ 和 $\mathrm{SnO}_{2}$ 用作锂离子电池负极的倍率性能对比; (b) $\mathrm{Sn}_{2} \mathrm{ONCN} / \mathrm{GN}$ 复合负极的倍率性能; (c) $\mathrm{Sn}_{2} \mathrm{ONCN} / \mathrm{GN}$ 复 合负极在 1 和 $2 \mathrm{~A} / \mathrm{g}$ 的循环性能; (d) $\mathrm{Sn}_{2} \mathrm{ONCN} / \mathrm{GN}$ 复合负极和近期报道的高性能 $\mathrm{SnO}_{2}$ 基负极的比容量、倍率性能对比;

(e) $\mathrm{SnO}_{2}$ 和(f) $\mathrm{Sn}_{2} \mathrm{ONCN}$ 电极脱嵌锂的反应机制; (g)商用 $\mathrm{LiCoO}_{2}$ 正极与 $\mathrm{TG} / \mathrm{MnNCN}$ 复合负极组成的全电池示意图, (h) 在不同倍率的充放电曲线, (i)倍率性能和循环稳定性 ${ }^{[57,61]}$

Fig. 5 (a) Comparison of rate capacities of $\mathrm{Sn}_{2} \mathrm{ONCN}$ and $\mathrm{SnO}_{2}$ anode materials for lithium-ion batteries; (b) Rate capacity of $\mathrm{Sn}_{2} \mathrm{ONCN} / \mathrm{GN}$ hybrid anode; (c) Cycling performance of $\mathrm{Sn}_{2} \mathrm{ONCN} / \mathrm{GN}$ hybrid anode at 1 and $2 \mathrm{~A} / \mathrm{g}$; (d) Comparison of the specific gravimetric rate capabilities among $\mathrm{Sn}_{2} \mathrm{ONCN} / \mathrm{GN}$ and the recently reported $\mathrm{SnO}_{2}$-based materials; Proposed reaction mechanisms of (e) $\mathrm{SnO}_{2}$ and (f) $\mathrm{Sn}_{2} \mathrm{ONCN}$ electrodes during charge/discharge; (g) Schematic diagram, (h) galvanostatic charge-discharge profiles at different rates, (i) rate capacities from $0.5 C$ to $20 C$ and cycling stability at $0.5 C$ rate of lithium-ion full cell assembled by commercial $\mathrm{LiCoO}_{2}$ cathode and TG/MnNCN hybrid anode ${ }^{[57,61]}$ 
$2.0 \mathrm{~A} / \mathrm{g}$ 高倍率循环 300 圈后, 比容量分别为 978 和 $758 \mathrm{mAh} / \mathrm{g}$, 稳定性突出 (图 5(c)) 。另外, $\mathrm{Sn}_{2} \mathrm{ONCN} / \mathrm{GN}$ 复合电极的倍率性能优于近期报道的 $\mathrm{SnO}_{2}$ 基负极(图 5(d))。

图 5(e, f) 对比了 $\mathrm{Sn}_{2} \mathrm{ONCN}$ 和 $\mathrm{SnO}_{2}$ 的电化学储 锂机制。 $\mathrm{SnO}_{2}$ 电极产生的 $\mathrm{Sn}$ 单质团聚为大颗粒而 失去活性，导致容量迅速衰减。而 $\mathrm{Sn}_{2} \mathrm{ONCN}$ 可以减 缓这个过程, 其准层状的晶体结构有利于锂离子的 传输和容忍体积膨胀。和易于迁移的 $\mathrm{O}^{2-}$ 离子相比, 各项异性的 $[\mathrm{N}=\mathrm{C}=\mathrm{N}]^{2-}$ 多原子离子难以迁移。 $[\mathrm{N}=\mathrm{C}=\mathrm{N}]^{2-}$ 网络有利于针定放电后产生的 $\mathrm{Li}_{2} \mathrm{O}$ 和 $\mathrm{Sn}$ 纳米颗粒，阻止形成 $\mathrm{Sn}$ 单质大颗粒，大大提高脱嵌 锂过程的可逆性, 因而 $\mathrm{Sn}_{2} \mathrm{ONCN}$ 具有优异的循环 稳定性。

金属氰胺化合物作为锂离子电池的负极材料已 有大量报道, 研究者们主要研究了单电极性能, 而 全电池性能鲜有报道。 $\mathrm{Li}$ 等 ${ }^{[57]}$ 构建了以自支撑三维 石墨烯负载 $\mathrm{MnNCN}$ 纳米颗粒 $(\mathrm{TG} / \mathrm{MnNCN})$ 为负极, 商用 $\mathrm{LiCoO}_{2}$ 为正极的全电池器件(图 5(g))。由于三 维石墨烯 $\mathrm{TG}$ 的高导电性和 $\mathrm{MnNCN}$ 与石墨烯 $\pi-\pi$ 共轭作用下的牢固负载, $\mathrm{TG} / \mathrm{MnNCN}$ 负极在不同面 密度 $\left(3 、 5\right.$ 和 $\left.10 \mathrm{mg} / \mathrm{cm}^{2}\right)$ 均能保持较高的质量比容量 和面积比容量。在 $10 \mathrm{mg} / \mathrm{cm}^{2}$ 的高载量下, $\mathrm{TG} / \mathrm{MnNCN}$ 负极仍然具有优异的倍率性能, 电流密 度 $0.5 \mathrm{~mA} / \mathrm{cm}^{2}$ 时, 面积比容量为 $9.8 \mathrm{mAh} / \mathrm{cm}^{2}$, 电流 密度高达 $50 \mathrm{~mA} / \mathrm{cm}^{2}$ 时, 面积比容量为 $3.5 \mathrm{mAh} / \mathrm{cm}^{2}$, 优于近期文献报道的负极材料。全电池器件在 $0.5 C$ 至 $20 C$ 倍率下均能稳定工作(图 5(h)), 容量分别为 133、110、80、63 和 $42 \mathrm{mAh} / \mathrm{g}$, 循环 120 圈后, 比 容量保持在 $124 \mathrm{mAh} / \mathrm{g}$ (图 5(i))。全电池的能量密度 和功率密度分别为 $343 \mathrm{Wh} / \mathrm{kg}$ 和 $8.7 \mathrm{~kW} / \mathrm{kg}$, 具有潜 在的应用前景。

根据文献报道，部分过渡金属氧胺化合物 $\left(\mathrm{FeNCN} 、 \mathrm{CoNCN} 、 \mathrm{NiNCN} 、 \mathrm{MnNCN}\right.$ 和 $\mathrm{Cr}_{2}(\mathrm{NCN})_{3}$ 等)的实际储锂容量高于理论容量。以 $\mathrm{MnNCN}$ 为例 ${ }^{[16]}$, 作为锂离子电池的负极材料, 其转换反应的理论容 量为 $565 \mathrm{mAh} / \mathrm{g}$, 而实验发现其可逆容量高达 $725 \mathrm{mAh} / \mathrm{g}$, 目前尚未对该现象有合理解释。这种实 际容量高于理论容量的异常现象, 凸显了金属氰胺 化合物储锂机理的复杂性。通过进一步的深入研究, 可以促进基于金属氰胺化合物的高能量密度电池的 设计和制备。

\section{2 钠离子电池}

某些金属氧胺化合物不仅表现出良好的储锂性 能, 而且在钠离子电池中也有突出表现。上述讨论
的 FeNCN, 也可以用于钠离子电池的负极材料 ${ }^{[18-19]}$ 。 Sougrati 等 ${ }^{[19]}$ 首先报道的 $\mathrm{FeNCN}$ 负极，展现出 $400 \mathrm{mAh} / \mathrm{g}$ 的高比容量, 200 圈循环后仍保持 $86 \%$, 比容量和循环稳定性均远高于 $\mathrm{FeO}$, 同时还具有较 高的倍率性能。最近, Cao 课题组 ${ }^{[56]}$ 通过高温热解草 酸铁铵和尿素的混合物制备了 $\mathrm{FeNCN}$ 独特的针状 结构。由于界面 $\mathrm{Fe}-\mathrm{C}$ 键的形成, 在进行多次电化学 循环的转化反应时, $\mathrm{Fe}-\mathrm{C}$ 键可以在 $\mathrm{FeNCN}$ 被还原 时抑制 $\mathrm{Fe}$ 纳米晶迁移，增强其循环稳定性。300 圈 循环后，容量保持率为 $79.9 \%$, 相对无界面键合的 对比样品, 稳定性显著提高, 且比容量提升了近 $20 \%$ 。通过进一步控制热解条件, Cao 课题组 ${ }^{[60]}$ 制备 了在碳祄底表面牢固负载的 FeNCN 晶粒。由于 FeNCN 的准层状六方结构, FeNCN 晶粒沿[001]方 向取向生长，暴露 $\{010\}$ 面。电流密度为 $0.2 、 0.5 、 1$ 、 2、5、10 和 $20 \mathrm{~A} / \mathrm{g}$ 时, FeNCN 负载碳电极的比容量 分别为 $686.6 、 671.2 、 660.1 、 657.0 、 623.6 、 544.5$ 和 $373.7 \mathrm{mAh} / \mathrm{g}$, 比容量和倍率性能均优于常见的 铁基负极(图 $6(\mathrm{a} \sim \mathrm{c})$ )。动力学分析表明, 电极容量主 要来自于表面控制的电容过程，比例高达 $87.7 \%$ 。 根据 $\mathrm{FeNCN}$ 的晶体结构, 通过计算 $\mathrm{Na}^{+}$沿不同晶向 的扩散势垒(图 6(d)), 相比[110]晶向，[001]晶向和 $<010>$ 晶向族有利于快速迁移和传输。由于 $\mathrm{Fe}-\mathrm{N}$ 共 价键的作用较强, 对 $\mathrm{Na}^{+}$的束缚较弱, $\mathrm{Na}^{+}$沿 $[001]$ 晶 向可在相邻的孔道迅速跃迁，而 $<010>$ 晶向族具有 合适的一维孔道结构，可促进 $\mathrm{Na}^{+}$传输。结合 FeNCN 晶粒的高度取向, $\mathrm{Na}^{+}$的高速传输可从晶格 层次扩展至晶粒层次，因而制备的 FeNCN 电极具 有优异的电化学储钠性能(图 6(d))。

\section{3 其它储能应用}

除了二次离子电池之外，金属氧胺化合物的电 化学储能应用还包括超级电容器和锂空气电池。 $\mathrm{Ji}$ 课题组 ${ }^{[63]}$ 报道了氰胺锌和石墨相氮化碳复合物 $\left(\mathrm{ZnNCN} / \mathrm{C}_{3} \mathrm{~N}_{4}\right)$ 用作水系超级电容器的电极材料, 组 装的非对称型超级电容器具有较高的能量密度 $(213 \mathrm{Wh} / \mathrm{kg})$; Yuan 等 ${ }^{[64]}$ 研究了纳米氧胺锰和氮掺杂 碳复合物 $(\mathrm{MnNCN} / \mathrm{NC})$ 的氧气析出/还原的催化性 能, 构建的锂空气电池具有比块体 $\mathrm{MnNCN}$ 和 $\mathrm{NC}$ 更加优异的循环稳定性。

\section{4 结论与展望}

本文总结了金属氰胺化合物的结构特点以及基 本物化性质，综述了金属氰胺化合物的固相、液相 合成方法及调控策略，介绍了金属氧胺化合物在电 

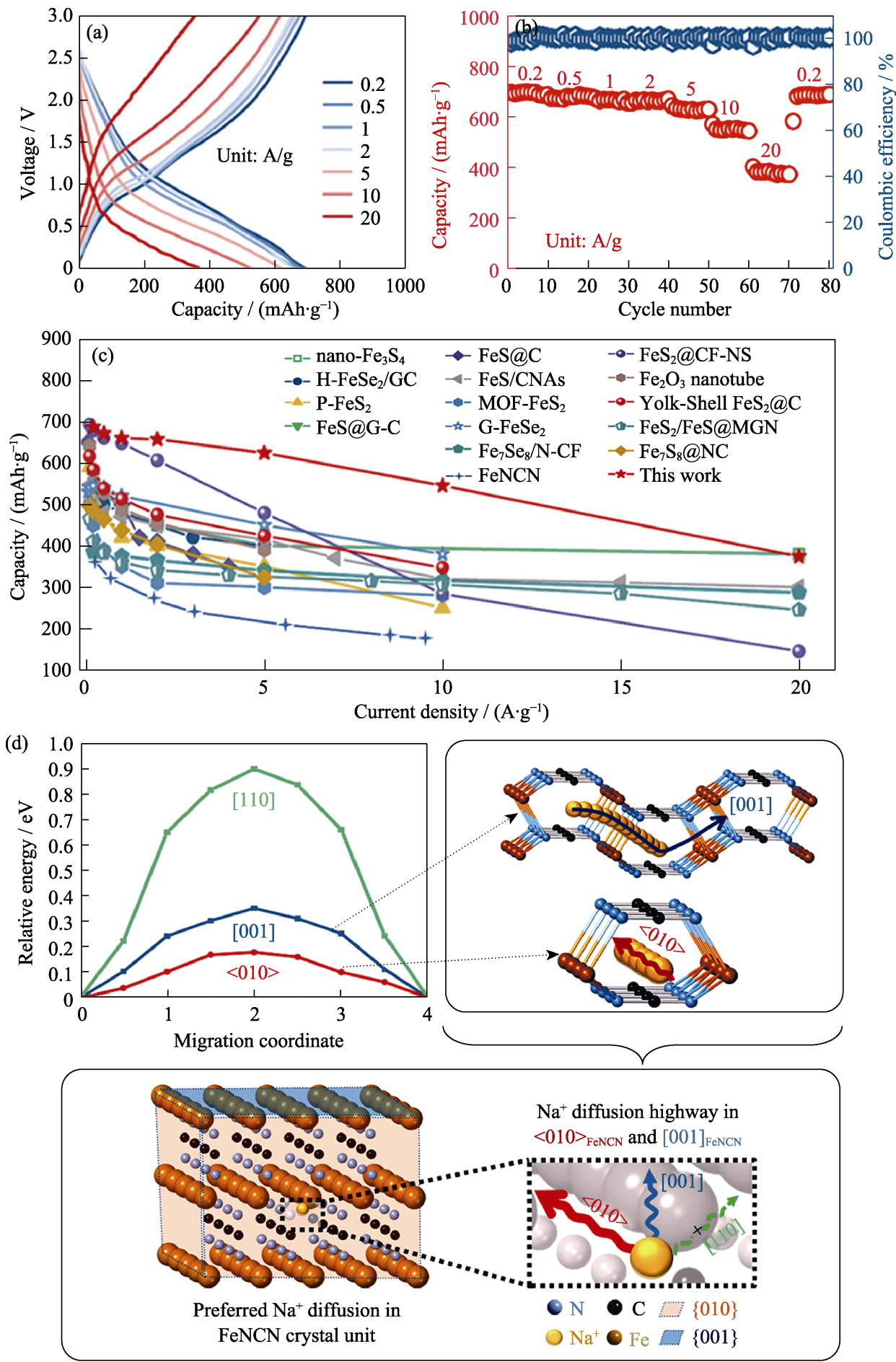

图 6 (a)FeNCN 用作钠离子电池负极在不同电流密度时的充放电曲线; (b)FeNCN 负极的倍率性能; (c)FeNCN 负极和近期报 道的高性能铁基无机材料负极的比容量、倍率性能对比; (d) Na ${ }^{+}$在 FeNCN 不同晶向的扩散势垒及传输机制 ${ }^{[60]}$

Fig. 6 (a) Galvanostatic charge-discharge profiles of FeNCN anode for sodium-ion battery at different current densities;

(b) Rate capacities at different current densities; (c) Comparison of the specific gravimetric rate capability among FeNCN and the recently reported iron-based inorganic materials; (d) Calculated $\mathrm{Na}^{+}$diffusion barrier along the three directions in FeNCN and the proposed diffusion highway ${ }^{[60]}$

Colourful figures are available on website

化学储能领域的代表性研究成果。在研究人员的持 续努力下，金属氧胺化合物的可控合成、物性探索 及应用前瞻均取得了重要进展, 但未来的研究工作 仍充满机遇与挑战。
首先，目前已报到的氰胺化合物不足一百种， 尚有许多由过渡金属元素组成的氰胺化合物未被合 成，尤其是高价金属 $\mathrm{Ti} 、 \mathrm{~V} 、 \mathrm{Mo} 、 \mathrm{~W}$ 等，随着合成 方法的改进和表征手段的升级，新化合物的设计制 
备、结构解析及物性探索将进一步推进，丰富金属 氰胺化合物的晶体学数据库及物化特性。

其次，针对金属氯胺化合物负极材料的下一步 研究, 本文有以下建议: (1)通过增加金属氰胺化合 物的种类，可以发现更多具有电化学活性的电极材 料并优化电化学储能性能; (2)结合先进表征技术和 理论模拟进一步深入研究电化学反应机理, 如厘清 部分过渡金属氰胺化合物的实际储锂容量高于理论 容量的原因, $[\mathrm{NCN}]^{2-}$ 的碳氮共轭 $\pi$ 键是否可以贡献 额外容量等; (3)拓展金属氰胺化合物的全电池应用, 提高能量密度和功率密度, 延长循环寿命等, 匹敌 甚至超过对应氧化物的器件性能。

另外, 纳米金属氯胺化合物在电化学储能等诸 多应用领域展现出比块体材料更优异的性能, 但其 纳米尺度材料制备的系统研究仍十分贵乏，如何控 制纳米晶的成核与生长，进而实现对其组分、尺寸、 形貌及结构的精细调控, 对于深入研究氰胺化合 物的构效关系以及获得高性能无机功能材料至关 重要。

\section{参考文献:}

[1] ZORKO A, JEGLIČ P, POTOČNIK A, et al. Unconventional magnetism in a nitrogen-containing analog of cupric oxide. Physical Review Letters, 2011, 107(4): 047208.

[2] DUVERNAY F, CHIAVASSA T, BORGET F, et al. Experimental study of water-ice catalyzed thermal isomerization of cyanamide into carbodiimide: implication for prebiotic chemistry. Journal of the American Chemical Society, 2004, 126(25): 7772-7773.

[3] Dehlinger U. XVII. Über die Raumgruppe von $\left(\mathrm{CN}_{2} \mathrm{H}_{2}\right)_{2}$ und die Kristallstruktur von $\mathrm{CaCN}_{2}$. Zeitschrift für KristallographieCrystalline Materials, 1927, 65: 286-290.

[4] BERGER U, SCHNICK W. Syntheses, crystal structures, and vibrational spectroscopic properties of $\mathrm{MgCN}_{2}, \mathrm{SrCN}_{2}$, and $\mathrm{BaCN}_{2}$. Journal of Alloys and Compounds, 1994, 206(2): 179-184.

[5] DOWN M G, HALEY M J, HUBBERSTEY P, et al. Synthesis of the dilithium salt of cyanamide in liquid lithium; X-ray crystal structure of $\mathrm{Li}_{2} \mathrm{NCN}$. Chemical Communications, 1978(2): 52-53.

[6] LIU X, DECKER A, SCHMITZ D, et al. crystal structure refinement of lead cyanamide and the stiffness of the cyanamide anion. Zeitschrift für Anorganische und Allgemeine Chemie, 2000, 626(1): $103-105$.

[7] DRONSKOWSKI R. $\operatorname{In}_{2.24}(\mathrm{NCN})_{3}$ and $\mathrm{NaIn}(\mathrm{NCN})_{2}$ : synthesis and crystal structures of new main group metal cyanamides. Zeitschrift für Naturforschung B, 1995, 50(8): 1245-1251.

[8] BECKER M, NUSS J, JANSEN M. Crystal structure and spectroscopic data of silver cyanamide. Zeitschrift für Naturforschung $B, 2000$, 55(5): 383-385.

[9] JIA B, SUN D, ZHAO W, et al. Metal cyanamides: openframework structure and energy conversion/storage applications. Journal of Energy Chemistry, 2021, 61: 347-367.

[10] WICKLEDER C. Thiocyanates as novel host lattices for emitting rare earth ions: luminescence of $\operatorname{Sr}(\mathrm{SCN})_{2}: \mathrm{Eu}^{2+}$. Chemistry of Materials, 2005, 17(5): 1228-1233.

[11] KRINGS M, MONTANA G, DRONSKOWSKI R, et al.
$\alpha$-SrNCN:Eu ${ }^{2+}-\mathrm{a}$ novel efficient orange-emitting phosphor. Chemistry of Materials, 2011, 23(7): 1694-1699.

[12] ZHAO W, LIU Y, LIU J, et al. Controllable synthesis of silver cyanamide as a new semiconductor photocatalyst under visible-light irradiation. Journal of Materials Chemistry A, 2013, 1(27): 7942-7948.

[13] RESSNIG D, SHALOM M, PATSCHEIDER J, et al. Photochemical and electrocatalytic water oxidation activity of cobalt carbodiimide. Journal of Materials Chemistry A, 2015, 3(9): 5072-5082.

[14] JIA B, ZHAO W, FAN L, et al. Silver cyanamide nanoparticles decorated ultrathin graphitic carbon nitride nanosheets for enhanced visible-light-driven photocatalysis. Catalysis Science \& Technology, 2018, 8(5): 1447-1453.

[15] SOUGRATI M T, ARAYAMPARAMBIL J J, LIU X, et al. Carbodiimides as energy materials: which directions for a reasonable future? Dalton Transactions, 2018, 47(32): 10827-10832.

[16] LIU C, ZHANG C, FU H, et al. Exploiting high-performance anode through tuning the character of chemical bonds for Li-ion batteries and capacitors. Advanced Energy Materials, 2017, 7(1): 1601127.

[17] LI Y, CAO C, ZHANG Q, et al. Nanorod bundle-like silver cyanamide nanocrystals for the high-efficiency photocatalytic degradation of tetracycline. RSC Advances, 2021, 11(17): 10235-10242.

[18] EGUIA-BARRIO A, CASTILLO-MARTINEZ E, LIU X, et al. Carbodiimides: new materials applied as anode electrodes for sodium and lithium ion batteries. Journal of Materials Chemistry A, 2016, 4(5): 1608-1611.

[19] SOUGRATI M T, DARWICHE A, LIU X, et al. Transition-metal carbodiimides as molecular negative electrode materials for lithium- and sodium-ion batteries with excellent cycling properties. Angewandte Chemie International Edition, 2016, 55(16): 5090-5095.

[20] KROTT M, HOUBEN A, MÜLLER P, et al. Determination of the magnetic structure of manganese carbodiimide with diffraction experiments using polarized neutrons. Physical Review B, 2009, 80(2): 024117.

[21] BECKER M, JANSEN M. Zinc cyanamide, $\mathrm{ZnCN}_{2}$. Acta Crystallographica Section C, 2001, 57(4): 347-348.

[22] LIU Q, LIU Y, DAI G, et al. Size-controllable synthesis of hierarchical copper carbodiimide microcrystals and their pronounced photoelectric response under visible light. Applied Surface Science 2015, 357: 745-749.

[23] LIU X, MÜLlER P, KROLL P, et al. Synthesis, structure determination, and quantum-chemical characterization of an alternate $\mathrm{HgNCN}$ polymorph. Inorganic Chemistry, 2002, 41(16): 4259-4265.

[24] LÖBER M, DOLABDJIAN K, STRÖBELE M, et al. Synthesis, structure, and electronic properties of $\mathrm{SnCN}_{2}$ and $\mathrm{Sn}_{4} \mathrm{Cl}_{2}\left(\mathrm{CN}_{2}\right)_{3}$. Inorganic Chemistry, 2019, 58(12): 7845-7851.

[25] KUBUS M, HEINICKE R, STRÖBELE M, et al. Synthesis of new structurally related cyanamide compounds $\operatorname{LiM}\left(\mathrm{CN}_{2}\right)_{2}$ where $\mathrm{M}$ is $\mathrm{Al}^{3+}, \mathrm{In}^{3+}$ or $\mathrm{Yb}^{3+}$. Materials Research Bulletin, 2015, 62: 37-41.

[26] DOLABDJIAN K, CASTRO C, MEYER H J. Layered carbodiimides $\mathrm{A}_{2} \mathrm{M}\left(\mathrm{CN}_{2}\right)_{3}$ with tetravalent cations $\mathrm{M}=\mathrm{Sn}, \mathrm{Zr}$, and $\mathrm{Hf}$. European Journal of Inorganic Chemistry, 2018(14): 1624-1630.

[27] CORKETT A J, KONZE P M, DRONSKOWSKI R. The ternary post-transition metal carbodiimide $\mathrm{SrZn}(\mathrm{NCN})_{2}$. Zeitschrift für Anorganische und Allgemeine Chemie, 2017, 643(21): 1456-1461.

[28] CORKETT A J, KONZE P M, DRONSKOWSKI R. Synthesis, crystal structure, and chemical-bonding analysis of $\mathrm{BaZn}(\mathrm{NCN})_{2}$. Inorganics, 2018, 6: 1-10.

[29] CORKETT A J, CHEN Z, BOGDANOVSKI D, et al. Band gap 
tuning in bismuth oxide carbodiimide $\mathrm{Bi}_{2} \mathrm{O}_{2} \mathrm{NCN}$. Inorganic Chemistry, 2019, 58(9): 6467-6473.

[30] DOLABDJIAN K, GÖRNE A L, DRONSKOWSKI R, et al. Tin(II) oxide carbodiimide and its relationship to SnO. Dalton Transactions, 2018, 47(38): 13378-13383.

[31] HASHIMOTO Y, TAKAHASHI M, KIKKAWA S, et al. Syntheses and crystal structures of trigonal rare-earth dioxymonocyanamides, $\mathrm{Ln}_{2} \mathrm{O}_{2} \mathrm{CN}_{2}(\mathrm{Ln}=\mathrm{Ce}, \mathrm{Pr}, \mathrm{Nd}, \mathrm{Sm}, \mathrm{Eu}, \mathrm{Gd})$. Journal of Solid State Chemistry, 1996, 125(1): 37-42.

[32] LI M, YUAN W, WANG J, et al. Syntheses and crystal structures of trigonal rare-earth dioxymonocyanamides, $\mathrm{Ln}_{2} \mathrm{O}_{2} \mathrm{CN}_{2}$ ( $\mathrm{Ln}=$ Dy, Ho, Er, Tm, Yb). Powder Diffraction, 2012, 22(1): 59-63.

[33] LI Z A, CHEN L X, CAI J R. First-principles study on electronic structure and half-metallic properties of $\mathrm{CoNCN}$ and NiNCN. Communications in Theoretical Physics, 2009, 52(4): 707-709.

[34] LIU X, KROTT M, MÜLLER P, et al. Synthesis, crystal structure, and properties of $\mathrm{MnNCN}$, the first carbodiimide of a magnetic transition metal. Inorganic Chemistry, 2005, 44(9): 3001-3003.

[35] TANG X, XIANG H, LIU X, et al. A ferromagnetic carbodiimide: $\mathrm{Cr}_{2}(\mathrm{NCN})_{3}$. Angewandte Chemie International Edition, 2010, 49(28): 4738-4742.

[36] JIA B, SUN D, ZHAO W, et al. Controllable conversion of $\mathrm{CdNCN}$ nanoparticles into various chalcogenide nanostructures for photo-driven applications. Chemistry-A European Journal, 2020, 26(35): 7955-7960.

[37] SRINIVASAN R, GLASER J, TRAGL S, et al. $\operatorname{LnCl}\left(\mathrm{CN}_{2}\right)$ with $\mathrm{Ln}=\mathrm{La}, \mathrm{Ce}$, and Pr: synthesis and structure of a new lanthanide chloride cyanamide related to the PbFCl-type structure. Zeitschrift für Anorganische und Allgemeine Chemie, 2005, 631(2/3): 479-483.

[38] NEUKIRCH M, TRAGL S, MEYER H J. Syntheses and structural properties of rare earth carbodiimides. Inorganic Chemistry, 2006, 45(20): 8188-8193.

[39] QIAO X, MA Z, LUO D, et al. Metathetic synthesis of lead cyanamide as a p-type semiconductor. Dalton Transactions, 2020, 49(40): 14061-14067.

[40] THOMAS A, FISCHER, A, GOETTMANN F, et al. Graphitic carbon nitride materials: variation of structure and morphology and their use as metal-free catalysts. Journal of Materials Chemistry, 2008, 18(41): 4893-4908.

[41] YAN X, YE Z, NING G, et al. Molten salt pyrolysis synthesis of magnetic FeNCN nanorods and their visible-light-driven photocatalytic properties. Applied Surface Science, 2020, 506: 145010.

[42] KROTT M, LIU X, MÜLLER P, et al. Synthesis and structure determination of $\mathrm{Co}(\mathrm{HNCN})_{2}$ and $\mathrm{Ni}(\mathrm{HNCN})_{2}$. Journal of Solid State Chemistry, 2007, 180(1): 307-312.

[43] KROTT M, LIU X, FOKWA B P T, et al. Synthesis, crystal structure determination and magnetic properties of two new transition-metal carbodiimides: $\mathrm{CoNCN}$ and NiNCN. Inorganic Chemistry, 2007, 46(6): 2204-2207.

[44] LIU X, STORK L, SPELDRICH M, et al. FeNCN and Fe(NCNH)2: synthesis, structure, and magnetic properties of a nitrogen-based pseudo-oxide and -hydroxide of divalent iron. Chemistry-A European Journal, 2009, 15(7): 1558-1561.

[45] LIU X, MÜLLER P, DRONSKOWSKI R. Synthesis and crystal structure of ammine copper(I) cyanamide, $\mathrm{Cu}_{4}(\mathrm{NCN})_{2} \mathrm{NH}_{3}$. Zeitschrift für Anorganische und Allgemeine Chemie, 2005, 631(6/7): 1071-1074.

[46] STORK L, LIU X, FOKWA B P T, et al. Crystal structure determination of thallium carbodiimide, $\mathrm{Tl}_{2} \mathrm{NCN}$. Zeitschrift für Anorganische und Allgemeine Chemie, 2007, 633(9): 1339-1342.

[47] JIA B, ZHAO W, SUN D, et al. Robust anion exchange realized in crystalline metal cyanamide nanoparticles. Chemistry of Materials,
2019, 31(22): 9532-9539.

[48] ZHAO W, PAN J, HUANG F. Nonaqueous synthesis of metal cyanamide semiconductor nanocrystals for photocatalytic water oxidation. Chemical Communications, 2018, 54(13): 1575-1578.

[49] KOZIEJ D, KRUMEICH F, NESPER R, et al. Nonaqueous liquid-phase synthesis of nanocrystalline metal carbodiimides. A proof of concept for copper and manganese carbodiimides. Journal of Materials Chemistry, 2009, 19(29): 5122-5124.

[50] EGUIA-BARRIO A, CASTILLO-MARTÍNEZ E, KLEIN F, et al. Electrochemical performance of $\mathrm{CuNCN}$ for sodium ion batteries and comparison with $\mathrm{ZnNCN}$ and lithium ion batteries. Journal of Power Sources, 2017, 367: 130-137.

[51] ARAYAMPARAMBIL J J, MANN M, FRAISSE B, et al. Cobalt carbodiimide as negative electrode for Li-ion batteries: electrochemical mechanism and performance. ChemElectroChem, 2019, 6(19): 5101-5108.

[52] ARAYAMPARAMBIL J J, MANN M, LIU X, et al. Electrochemical evaluation of $\mathrm{Pb}, \mathrm{Ag}$, and $\mathrm{Zn}$ cyanamides/carbodiimides. ACS Omega, 2019, 4(2): 4339-4347.

[53] ARAYAMPARAMBIL J J, CHEN K, IADECOLA A, et al. Reversible high capacity and reaction mechanism of $\mathrm{Cr}_{2}(\mathrm{NCN})_{3}$ negative electrodes for Li-ion batteries. Energy Technology, 2020, 8(3): 1901260.

[54] BRAUN C, MEREACRE L, HUA W, et al. $\mathrm{SnCN}_{2}$ : a carbodiimide with an innovative approach for energy storage systems and phosphors in modern LED technology. ChemElectroChem, 2020, 7(22): 4550-4561.

[55] CHEN K, FEHSE M, LAURITA A, et al. Quantum-chemical study of the FeNCN conversion-reaction mechanism in lithium- and sodium-ion batteries. Angewandte Chemie International Edition, 2020, 59(9): 3718-3723.

[56] GUO P, CAO L, WANG R, et al. In situ construction of "anchor-like" structures in FeNCN for long cyclic life in sodiumion batteries. Advanced Functional Materials, 2020, 30(17): 2000208.

[57] LI T, ZHAO W, BI H, et al. Tubular graphene-supported nanoparticulate manganese carbodiimide as a free-standing high-energy and high-rate anode for lithium ion batteries. Journal of Power Sources, 2020, 467: 228252.

[58] HE W, LI H, LONG B, et al. One-step synthesis of $\mathrm{ZnNCN}$ nanoparticles with adjustable composition for an advanced anode in lithium ion battery. ACS Applied Energy Materials, 2021, 4(5): 4290-4296.

[59] CHEN K, LUO D, DRONSKOWSKI R. Exploring the possible anionic redox mechanism in Li-rich transition-metal carbodiimides. Journal of Physical Chemistry C, 2021, 125(16): 8479-8487.

[60] LI J, WANG R, GUO P, et al. Realizing fast charge diffusion in oriented iron carbodiimide structure for high-rate sodium-ion storage performance. ACS Nano, 2021, 15(4): 6410-6419.

[61] LÜ Z, DONG W, JIA B, et al. Flexible yet robust framework of tin(II) oxide carbodiimide for reversible lithium storage. Chemistry-A European Journal, 2021, 27(8): 2717-2723.

[62] ZHAO S, SEWELL C D, LIU R, et al. $\mathrm{SnO}_{2}$ as advanced anode of alkali-ion batteries: inhibiting Sn coarsening by crafting robust physical barriers, void boundaries, and heterophase interfaces for superior electrochemical reaction reversibility. Advanced Energy Materials, 2020, 10(6): 1902657.

[63] SHEN J, CHEN X, WANG P, et al. Electrochemical performance of zinc carbodiimides based porous nanocomposites as supercapacitors. Applied Surface Science, 2021, 541: 148355.

[64] YUAN M, ZHANG S, LIN L, et al. Manganese carbodiimide nanoparticles modified with $\mathrm{N}$-doping carbon: a bifunctional cathode electrocatalyst for aprotic $\mathrm{Li}_{2} \mathrm{O}_{2}$ battery. ACS Sustainable Chemistry \& Engineering, 2019, 7(20): 17464-17473. 